\title{
Weighted Sum Secrecy Rate Maximization using Intelligent Reflecting Surface
}

\author{
Hehao Niu, Zheng Chu, Member, IEEE, Fuhui Zhou, Senior Member, IEEE, \\ Zhengyu Zhu, Member, IEEE, Miao Zhang, Member, IEEE, \\ and Kai-Kit Wong, Fellow, IEEE
}

\begin{abstract}
This paper aims to investigate the benefit of using intelligent reflecting surface (IRS) in multi-user multiple-input single-output (MU-MISO) systems, in the presence of eavesdroppers. We maximize the
\end{abstract}

This work was supported by the National Natural Science Foundation of China under Grant 61671454 and 61901490.

H. Niu is with the Institute of Electronic Countermeasure, National University of Defense Technology, Hefei, 230037, China, (e-mail: niuhaonupt@foxmail.com).

Z. Chu is with the 5GIC \& 6GIC, Institute for Communication Systems (ICS), University of Surrey, Guildford GU2 7XH, U.K., (e-mail: andrew.chuzheng7@gmail.com).

F. Zhou is with the College of Electronic and Information Engineering, Nanjing University of Aeronautics and Astronautics, Nanjing, 210000. China, and also with the Key Laboratory of Dynamic Cognitive System of Electromagnetic Spectrum Space (Nanjing University of Aeronautics and Astronautics) and with the Ministry of Industry and Information Technology, Nanjing 211106, China, (e-mail: zhoufuhui@ ieee.org).

Z. Zhu is with the School of Information Engineering, Zhengzhou University, Zhengzhou 450001, China, (e-mail: iezyzhu@zzu.edu.cn).

M. Zhang is with the School of Information Science and Engineering, Chongqing Jiaotong University, Chongqing, 400074, China, (e-mail: msczz@foxmail.com).

K.-K. Wong is with the Department of Electronic and Electrical Engineering, University College London, London, WC1E 6BT, U. K., (email: kai-kit.wong@ucl.ac.uk). 
weighted sum secrecy rate by jointly designing the secure beamforming (BF), the artificial noise (AN), as well as the phase shift of the IRS. An alternating optimization (AO) method is proposed to deal with the formulated non convex problem. In particular, the secure beamforming and AN jamming matrix are optimally designed via the successive convex approximation (SCA) approach for given phase shift, which can be derived by considering the alternating direction method of multiplier (ADMM) and element-wise block coordinate decent (EBCD) methods. Finally, simulation results are presented to show the benefit of the IRS in terms of improving the secrecy performance, when compared to other methods.

\section{Index Terms}

Intelligent reflecting surface (IRS), secure transmission, alternating optimization, alternating direction method of multiplier (ADMM), element-wise block coordinate decent (EBCD).

\section{INTRODUCTION}

With the development of the fifth-generation $(5 \mathrm{G})$ and beyond wireless networks, the realization of high speed information transmission with lower energy consumption has become a challenging task [1]. Conventionally, this challenge is met by utilization of renewable energy, low energy consumption devices, and efficient signal processing or resource allocation techniques [2]. However, these methods may be difficult to realize when considering the hardware limitation [3]. Thus, a new technique is needed to achieve energy-efficient and cost-effective wireless communication.

Recently, intelligent reflecting surface (IRS) has emerged to be a promising solution [4]. Specifically, IRS, is a planar array which is composed of a large number of reflecting units, and controlled by a programmable IRS controller [5]. Particularly, these reflecting units are generally made of small size, low-cost elements which can alter the phase of the received signal [6]. Without the need of encoding, decoding, or re-transmission operation, the power consumption of IRS is potentially much less than that for the commonly used active transmitters or relays [7]. For this reason, IRS has aroused great research interests in recent years. 
Specifically, in [8], the authors investigated the joint transmit beamforming (BF) and reflecting coefficient (RC) optimization in downlink multiple-input single-output (MISO) systems assisted by the IRS, where a transmit power cost function was minimized. In [9], the authors investigated the joint BF and RC design for an IRS-assisted MISO network with simultaneous wireless information and power transfer (SWIPT), where the harvested power at the energy receiver is maximized. In [10], the authors investigated the IRS-aided design in multi-user MISO (MUMISO) downlink channels, where a Lagrange dual method was proposed to maximize the weighted sum rate among the users. In [11], the authors proposed a robust framework in IRSaided MISO channels with imperfect channel state information (CSI), where a penalty constraint convex concave procedure (CCCP) method was proposed to optimize the RC. Recently in [12], the authors investigated the joint BF and phase shifts optimization with discrete RC. While in [13], the authors studied the BF design for an IRS-aided MISO networks with practical hardware impairments for the transceiver, where a closed-form optimal solution was obtained.

Furthermore, the IRS-aided transmission design has been investigated in the multiple-input multiple-output (MIMO) system in [14], the multi-group multi-cast network in [15], and the multi-cell network in [16], respectively. Besides, IRS-aided transmission has been considered to work with other emerging techniques such as deep learning [17], cognitive radio (CR) network [18], wireless powered communication network (WPCN) [19], full-duplex (FD) communication [20], non-orthogonal multiple access (NOMA) [21], and directional modulation (DM) [22], respectively. Summarizing the literature, the manifold optimization [10], the majorizationminimization (MM) method [13], and the semi-definite programming (SDP) with Gaussian randomization (GR) [18] are commonly used to optimize the RC.

The above work mainly focused on the scenario with one IRS or multiple distributed IRSs each independently serving its associated users in the vicinity. On the other hand, several recent works have studied the joint BF design among multiple IRSs. Specifically, in [23], the authors investigated the cooperative passive BF design in a double-IRS-aided network, and was extended by considering channel estimation in [24]. While in [26], the authors studied the cooperative 
passive BF design in a double-IRS-assisted MIMO uplink network. Recently in [25], the authors studied the multi-hop signal reflection in a multi-IRS-aided network.

Security is an important field in 5G and future wireless networks. Traditionally, security is provided by the network layer cryptographic method, but key distribution and management is difficult. Thus, physical layer security (PLS) techniques, which explore the randomness of wireless channel to improve the security, have attracted great attention in recent years [27].

Since IRS can adaptively adjust the amplitude and phase of the received signal, the reflected signal can be enhanced or weakened at different receivers. This virtue makes IRS appealing to the PLS design. In fact, the secrecy performance can be improved via designing passive BF of the IRS [28]. Recently, the IRS-aided secure wireless communication was investigated. Specifically, the secrecy transmission in IRS-aided MISO channel was investigated in [29] and [30], while the aim was to maximize the secrecy rate and minimize the transmit power, respectively. Also in [31], the authors investigated the IRS-aided secure transmission in MIMO wiretap channels, where a MM based optimization method was proposed to maximize the achievable secrecy rate. Moreover, in [32], the authors investigated the robust secrecy design in the IRS-aided downlink MISO network, where a transmit power minimization design was solved by the penalty CCCP method. Actually, the artificial noise (AN) aided method can further improve the secrecy performance, at the cost of transmit power consumption intended for the legitimate receiver. Thus, the AN aided method may become the performance bottleneck since the transmit power is commonly limited.

Fortunately, the IRS technique can be exploited to overcome the challenge. Specifically, in [33] and [34], the authors investigated the IRS-aided secure MIMO transmission, where a MM based method and a two layer algorithm were proposed to maximize the secrecy rate, respectively. In [35], the authors investigated the IRS-aided PLS design considering statistical CSI, where a sample average approximation method was proposed to maximize the achievable secrecy rate. In [36], the authors investigated the IRS-aided transmission without the knowledge of Eve's CSI, where a null space based AN method was proposed to minimize the transmit power. Recently, in [37], the authors studied the secure multi-group multi-cast network via IRS, where a SDP- 
GR method was proposed to design the BF. Besides, to reduce the computational complexity, a second-order cone programming (SOCP)-based method was studied. While in [38], the authors studied the double-IRS-aided secure transmission with inter-surface signal reflection, where a product Riemmanian manifold method was proposed to maximize the secrecy rate.

However, these works mainly focused on the joint BF design via various optimization methods. The joint design of active and passive BF as well as AN in secrecy multi-user networks is not well understood. Motivated by this, we investigate the IRS-aided secure design in the MUMISO downlink channel. Specifically, we aim to obtain the maximum weighted sum secrecy rate (WSSR), by jointly optimizing the BF vector, the AN covariance, and the RC at the IRS, under the practical constraints on the transmit power and the RC. The formulated problem is non convex, due to the unit-modulus constraint for the RC. To overcome this obstacle, we reformulate the non convex problem by linearizing the objective function. Then, an alternating optimization (AO) approach is proposed, in which the BF and $\mathrm{AN}$ are solved by the successive convex approximation (SCA) method while the RC is solved by the direction method of multipliers (ADMM) method and the element-wise block coordinate decent (EBCD) method. Finally, simulation results assess the performance of the proposed design.

Although [32] and [37] have studied the joint BF and AN optimization in IRS-aided secure network, there exist several difference of our work with these two works:

1) [32] assumed that the Eve are equipped with multiple antennas and the directly links are negligible, while we focus on single-antenna Eve scenario and both the direct link and the cascaded links are considered. Besides, [37] studied the secrecy multi-group multi-cast network, while we assume a multi-user multi-cast channel, e.g., there exits only one Bob in each group. Thus, the considered system model is different.

2) [32] proposed a penalty-based SDP method to maximize the achievable rate at the Bobs, while keeping the channel capacity of the Eve's link below a predefined threshold. Then, [37] proposed a SDP method and a SOCP method to minimize the transmit power under the secrecy rate constraint. On the other hand, we treat the WSSR as the objective and the 
proposed method mainly based on ADMM and EBCD, and not involved with the penaltybased approximation method or the GR method. Thus, the optimization problem and the corresponding technique way in our work is different with these works.

We summarize our main contributions as follows:

1) We aim to maximize the WSSR by jointly optimizing the active BF, the AN covariance and the RC at the IRS. Two kind of constraints on the reflecting elements, e.g., the continuous $\mathrm{RC}$ and discrete RC are considered, respectively. The formulated problem is difficult to solve, since the objective function is not concave and the optimization variables are highlycoupled. To tackle this, a newly linearization technique is proposed to reformulate the objective function into a more solvable formulation.

2) The AO algorithm is used to obtain the solution in an alternating way. Firstly, with the fixed phase shifts of the IRS, the optimal BF vector and AN covariance are obtained via the SCA method. Then, with the fixed BF vector and AN covariance, we formulate a quadratically constrained quadratic program (QCQP) with respect to (w.r.t.) the IRS phase shifts. However, the non convex unit modulus constraints makes the problem difficult to handle directly. To address this, the ADMM and EBCD methods are proposed, where the phase shifts can be obtained in closed form iteratively. Henceforth, the optimal solution to the original problem can be obtained efficiently.

3) The proposed method can be directly applied to the discrete RC and multiple IRSs case. In addition, the complexity comparisons of the proposed method and other methods is discussed, and the convergence behaviour of the proposed method is analyzed and examined. Both the outer and inner algorithms converge in a few number of iterations.

4) The simulation results confirm that IRS can improve the secrecy performance of an ANaided MISO system, provided the phase shifts are appropriately optimized. In addition, we conclude several insights. First, the IRS plays a more important role than AN in improving the security. Also, the main effect of IRS is to enhance the desired signal power at the Bobs, and reduce the desired signal power at the Eves. Besides, IRS should be deployed 
near the BS or Bobs to improve the secrecy performance, while multiple small IRSs can obtain better performance than a large IRS when each Bob-Eve group is scattered in a relatively large region.

The remainder of this paper is organized as follows. Section II provides the signal model of a downlink secrecy MU-MISO system assisted by one IRS, where the WSSR maximization (WSSRM) problem is formulated. The WSSRM design is reformulated in Section III, where an AO algorithm is proposed. In Section IV, we discuss the application scenarios of the proposed method. In Section V, simulation results are given to verify the algorithm performance. Section VI concludes this paper.

Notations: In this paper, boldface lowercase and uppercase letters denote vectors and matrices, respectively. The conjugate, transpose, conjugate transpose, and trace of matrix $\mathbf{A}$ are denoted as $\mathbf{A}^{\dagger}, \mathbf{A}^{T}, \mathbf{A}^{H}$, and $\operatorname{Tr}(\mathbf{A})$, respectively. $\mathbf{a}=\operatorname{vec}(\mathbf{A})$ stacks the columns of matrix $\mathbf{A}$ into a vector a. $\mathbf{A} \succeq \mathbf{0}$ indicates that $\mathbf{A}$ is a positive semi-definite matrix. \|\| denotes the Euclidean norm of a vector or the Frobenius of a matrix. $\odot$ denotes the element-wise product. $\operatorname{diag}(\mathbf{a})$ represents a diagonal matrix with a on the main diagonal. $\mathbf{I}$ is an identity matrix with proper dimension. $\mathcal{C N}(\mathbf{0}, \mathbf{I})$ denotes a circularly symmetric complex Gaussian random vector with mean $\mathbf{0}$ and covariance I. $\Re\{a\}$ denotes the real part of a complex variable $a$.

\section{System Model AND Problem Formulation}

In this section, we discuss the secrecy IRS-aided MU-MISO downlink system model. Then, a WSSRM design is proposed. It should be noted that due to the severe "distance-product" power loss and diffraction with multiple reflection [8], the power of signals reflected twice or more is much smaller than that of the signal reflected only once, thus can be omitted.

\section{A. IRS Model}

The phase shift matrix of IRS is denoted by $\boldsymbol{\Theta}=\sqrt{\eta} \operatorname{diag}\left(\theta_{1}, \ldots, \theta_{M}\right)$, with $\theta_{m}$ denoting the $\mathrm{RC}$ of the $m$-th reflection element. Without loss of generality, the reflection efficiency $\eta$ is 
assumed to be $\eta=1$. Besides, we make the following assumptions for the feasible set of the RC. In this work, since a narrow band system is considered, the resonance effects is not considered. In fact, in practical reflect model, the reflection amplitude is a function of the phase shifts [39], which is more complex.

1) Continuous phase shifter: In the continuous $\mathrm{RC}$ case, only the phase of the received signal is changed, while the strength of the reflection signal from each reflection element is maximized, e.g., $\left|\theta_{m}\right|^{2}=1$. Since $\theta_{m}$ can be optimized to any possible phase, we have $\mathcal{F}_{1}=$ $\left\{\theta_{m} \mid \theta_{m}=e^{j \phi_{m}}, \phi_{m} \in[0,2 \pi)\right\}$.

2) Discrete phase shifter: In the discrete RC case, the reflection element only takes finite values. Similarly with the model in [12], we assume that $\theta_{m}$ just has $\tau$ discrete values, which are equally spaced on the circle $\theta_{m}=e^{j \phi_{m}}$, i.e., $\mathcal{F}_{2}=\left\{\theta_{m} \mid \theta_{m}=e^{j \phi_{m}}, \phi_{m} \in\left\{0, \frac{2 \pi}{\tau}, \cdots, \frac{2 \pi(\tau-1)}{\tau}\right\}\right\}$.

In practice, due to the hardware limitation, it is costly to achieve continuous $\mathrm{RC}$ on the reflection elements. Hence, utilizing the discrete $\mathrm{RC}$ on the reflection elements is more realistic. However, it is meaningful to investigate the continuous RC design, since the optimization method in the continuous RC case is helpful to the discrete RC case.

\section{B. System Model}

As shown in Fig. 1, we consider a narrow band downlink MU-MISO system which consists of one base station (BS), one IRS, $K$ legitimate users (Bobs) and $K$ eavesdroppers (Eves), with the sets for Bobs denoted as $\left\{B_{1}, \ldots, B_{K}\right\}$, and for Eves denotes as $\left\{E_{1}, \ldots, E_{K}\right\}$, respectively. The BS and the IRS are equipped with $N$ antennas and $M$ reflection elements, respectively, while all the Bobs and Eves are single antenna nodes. We denote $\mathbf{G} \in \mathbb{C}^{M \times N}, \mathbf{h}_{d, k} \in \mathbb{C}^{M \times 1}$, $\mathbf{h}_{r, k} \in \mathbb{C}^{N \times 1}, \mathbf{h}_{d, e, k} \in \mathbb{C}^{M \times 1}$, and $\mathbf{h}_{r, e, k} \in \mathbb{C}^{N \times 1}$ as the channels between BS and IRS, between BS and the $k$-th Bob, between IRS and the $k$-th Bob, between BS and the $k$-th Eve, between IRS and the $k$-th Eve, for $k=1, \ldots, K$, respectively. In addition, the controller shown in Fig. 1 is utilized to coordinate the BS and IRS for CSI acquisition and information transmission.

In this paper, all CSI is assumed to be perfectly obtained at the BS and the IRS, which 


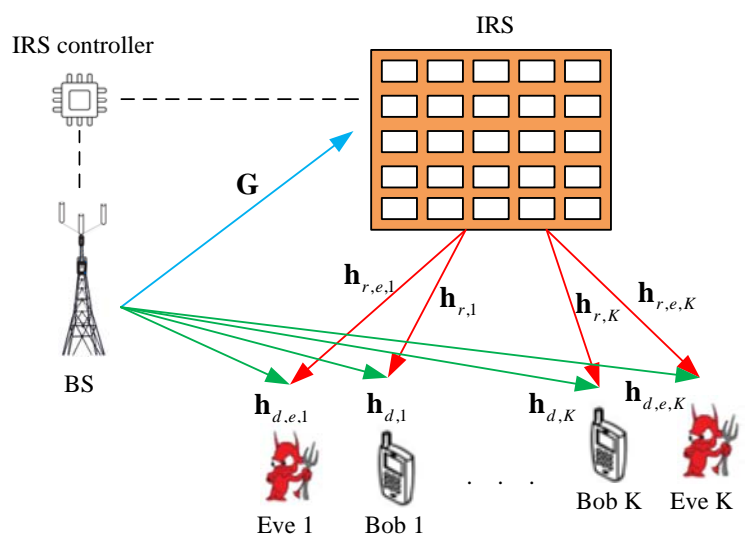

Fig. 1. The IRS-aided secure MISO system model.

is due to the fact that we aim to obtain an upper bound of the secrecy performance in the IRS network, which is similar to [28]-[31]. Besides, several channel estimation techniques have been investigated for obtaining the CSI such as the brute-force method and the semi-passive IRS method in [10], the discrete Fourier transform (DFT)-matrix quantization in [42], and the compressed sensing method in [43], e.t.c., which makes the estimation and acquirement of the CSI become practical.

The BS sends $K$ independent data streams for each Bob in the same frequency band simultaneously, under the thread of several Eves. Furthermore, we assume that the $K$ Eves are trying to eavesdrop any of the data streams, independently. Let us denote $s_{k}$ as the confidential message intended to the $B_{k}$, with $\mathbb{E}\left\{\left|s_{k}\right|^{2}\right\}=1$. Since AN is injected to degrade the Eves, the transmitted signal is given by $\mathbf{x}=\sum_{k=1}^{K} \mathbf{w}_{k} s_{k}+\mathbf{v}$, where $\mathbf{w}_{k} \in \mathbb{C}^{N \times 1}$ denotes the $\mathrm{BF}$ vector intent to the $k$-th Bob, and $\mathbf{v} \in \mathbb{C}^{N \times 1}$ denotes the $\mathrm{AN}$ vector.

Thus, the received signals at the $k$-th Bob and the $k$-th Eve are respectively, given by

$$
\begin{aligned}
& y_{k}=\left(\mathbf{h}_{d, k}^{H}+\mathbf{h}_{r, k}^{H} \boldsymbol{\Theta}^{H} \mathbf{G}\right)\left(\sum_{k=1}^{K} \mathbf{w}_{k} s_{k}+\mathbf{v}\right)+n_{k}, \\
& y_{e, k}=\left(\mathbf{h}_{d, e, k}^{H}+\mathbf{h}_{r, e, k}^{H} \boldsymbol{\Theta}^{H} \mathbf{G}\right)\left(\sum_{k=1}^{K} \mathbf{w}_{k} s_{k}+\mathbf{v}\right)+n_{e, k},
\end{aligned}
$$

where $n_{k}$ is the zero-mean additive Gaussian noise at the $k$-th Bob with variance $\sigma_{k}^{2}$, and $n_{e, k}$ is the zero-mean additive Gaussian noise at the $k$-th Eve with variance $\sigma_{e, k}^{2}$, respectively. 
In fact, by denoting $\boldsymbol{\theta}=\left[\theta_{1}, \ldots, \theta_{M}\right]^{T}$, we have

$$
\begin{aligned}
& \left(\mathbf{h}_{d, k}^{H}+\mathbf{h}_{r, k}^{H} \boldsymbol{\Theta}^{H} \mathbf{G}\right) \mathbf{w}_{k}=\hat{\boldsymbol{\theta}}^{H} \mathbf{H}_{k} \mathbf{w}_{k}, \\
& \left(\mathbf{h}_{d, e, k}^{H}+\mathbf{h}_{r, e, k}^{H} \boldsymbol{\Theta}^{H} \mathbf{G}\right) \mathbf{w}_{k}=\hat{\boldsymbol{\theta}}^{H} \mathbf{H}_{e, k} \mathbf{w}_{k},
\end{aligned}
$$

where $\hat{\boldsymbol{\theta}}=\left[\boldsymbol{\theta}^{H}, 1\right]^{H}, \mathbf{H}_{k}=\left[\operatorname{diag}\left(\mathbf{h}_{r, k}^{H}\right) \mathbf{G}, \mathbf{h}_{d, k}^{H}\right]^{T}$, and $\mathbf{H}_{e, k}=\left[\operatorname{diag}\left(\mathbf{h}_{r, e, k}^{H}\right) \mathbf{G}, \mathbf{h}_{d, e, k}^{H}\right]^{T}$, respectively.

Thus, with perfect CSI of Bobs and Eves, the achievable secrecy rate for the $k$-th Bob is

$$
R_{s, k}=\ln \left(1+\gamma_{k}\right)-\ln \left(1+\gamma_{e, k}\right)
$$

where $\gamma_{k}$ and $\gamma_{e, k}$ denote the signal-to-interference-plus-noise ratio (SINR) at the $k$-th Bob and the $k$-th Eve, which are given by

$$
\begin{gathered}
\gamma_{k}=\frac{\left|\hat{\boldsymbol{\theta}}^{H} \tilde{\mathbf{H}}_{k} \mathbf{w}_{k}\right|^{2}}{\sum_{i=1, i \neq k}^{K}\left|\hat{\boldsymbol{\theta}}^{H} \tilde{\mathbf{H}}_{k} \mathbf{w}_{i}\right|^{2}+\left|\hat{\boldsymbol{\theta}}^{H} \tilde{\mathbf{H}}_{k} \mathbf{v}\right|^{2}+1}, \\
\gamma_{e, k}=\frac{\left|\hat{\boldsymbol{\theta}}^{H} \tilde{\mathbf{H}}_{e, k} \mathbf{w}_{k}\right|^{2}}{\sum_{i=1, i \neq k}^{K}\left|\hat{\boldsymbol{\theta}}^{H} \tilde{\mathbf{H}}_{e, k} \mathbf{w}_{i}\right|^{2}+\left|\hat{\boldsymbol{\theta}}^{H} \tilde{\mathbf{H}}_{e, k} \mathbf{v}\right|^{2}+1},
\end{gathered}
$$

where $\tilde{\mathbf{H}}_{k}=\mathbf{H}_{k} \sigma_{k}^{-1}$ and $\tilde{\mathbf{H}}_{e, k}=\mathbf{H}_{e, k} \sigma_{e, k}^{-1}$.

\section{Problem Formulation}

In this work, we aim to jointly design the $\mathrm{BF} \mathbf{w}_{k}$, the $\mathrm{AN} \mathbf{v}$, and the phase shift $\hat{\boldsymbol{\theta}}$ to achieve the maximum WSSR. Mathematically, the problem is formulated as

$$
\begin{aligned}
\max _{\mathbf{w}_{k}, \mathbf{v}, \hat{\theta}} & R_{s} \triangleq \sum_{k=1}^{K} \varphi_{k}\left(\ln \left(1+\gamma_{k}\right)-\ln \left(1+\gamma_{e, k}\right)\right) \\
\text { s.t. } & \sum_{k=1}^{K}\left\|\mathbf{w}_{k}\right\|^{2}+\|\mathbf{v}\|^{2} \leq P_{T}, \\
& \left|\hat{\theta}_{m}\right|=1, \forall m \in \mathcal{M}, \hat{\theta}_{M+1}=1,
\end{aligned}
$$

where $\varphi_{k}\left(0 \leq \varphi_{k} \leq 1, \sum_{k=1}^{K} \varphi_{k}=1\right)$ is the weighted factor for the $k$-th Bob and $P_{T}$ is the transmit power budget. 


\section{JOINT BF, AN AND RC DESIGN}

Here, we mainly focus on the design for the continuous RC case, since the proposed method can be used to the discrete RC case. In fact, (5) is hard to solve, since (5a) is not concave and the constraint (5c) is not convex. To find the solution of (5) efficiently, we linearize (5a) and decompose (5) into two subproblems. Then, the corresponding methods to the subproblems are developed.

\section{A. BF and AN Covariance Optimization}

Firstly, we propose an SCA method to optimize the BF and AN with the fixed phase shift coefficient $\hat{\boldsymbol{\theta}}$. The main idea is to reformulate an approximated problem of (5) around the given point $\left\{\mathbf{w}_{k}^{q}, \mathbf{v}^{q}, \hat{\boldsymbol{\theta}}^{q}\right\}$ at the $q$-th iteration. Here, we use the following result in [44] to transform the rate w.r.t. the $k$-th Bob into a solvable formulation, given by

where $\{\bar{\alpha}, \bar{\beta}\}$ is a fixed point.

$$
\ln \left(1+\frac{|\alpha|^{2}}{\beta}\right) \geq \ln \left(1+\frac{|\bar{\alpha}|^{2}}{\bar{\beta}}\right)-\frac{|\bar{\alpha}|^{2}}{\bar{\beta}}+\frac{2 \Re\{\bar{\alpha} \alpha\}}{\bar{\beta}}-\frac{|\bar{\alpha}|^{2}\left(\beta+|\alpha|^{2}\right)}{\bar{\beta}\left(\bar{\beta}+|\bar{\alpha}|^{2}\right)},
$$

Based on the above equation, the information rate for the $k$-th Bob can be approximated as

$$
\ln \left(1+\gamma_{k}\right) \geq \ln \left(1+\frac{\left|x_{k}^{q}\right|^{2}}{y_{k}^{q}}\right)-\frac{\left|x_{k}^{q}\right|^{2}}{y_{k}^{q}}+2 \frac{\Re\left\{x_{k}^{q} x_{k}\right\}}{y_{k}^{q}}-\frac{\left|x_{k}^{q}\right|^{2}\left(y_{k}+\left|x_{k}\right|^{2}\right)}{y_{k}^{q}\left(y_{k}^{q}+\left|x_{k}^{q}\right|^{2}\right)},
$$

where $x_{k}^{q}=\left(\hat{\boldsymbol{\theta}}^{q}\right)^{H} \tilde{\mathbf{H}}_{k} \mathbf{w}_{k}^{q}, x_{k}=\left(\hat{\boldsymbol{\theta}}^{q}\right)^{H} \tilde{\mathbf{H}}_{k} \mathbf{w}_{k}, y_{k}^{q}=\sum_{i=1, i \neq k}^{K}\left|\left(\hat{\boldsymbol{\theta}}^{q}\right)^{H} \tilde{\mathbf{H}}_{k} \mathbf{w}_{i}^{q}\right|^{2}+\left|\left(\hat{\boldsymbol{\theta}}^{q}\right)^{H} \tilde{\mathbf{H}}_{k} \mathbf{v}^{q}\right|^{2}+1$, and $y_{k}=\sum_{i=1, i \neq k}^{K}\left|\left(\hat{\boldsymbol{\theta}}^{q}\right)^{H} \tilde{\mathbf{H}}_{k} \mathbf{w}_{i}\right|^{2}+\left|\left(\hat{\boldsymbol{\theta}}^{q}\right)^{H} \tilde{\mathbf{H}}_{k} \mathbf{v}\right|^{2}+1$.

Next, we transform the information rate for the $k$-th Eve into a new formulation. We firstly rewrite the term as

$$
-\ln \left(1+\gamma_{e, k}\right)=\ln \left(1+\sum_{i=1, i \neq k}^{K}\left|\left(\hat{\boldsymbol{\theta}}^{q}\right)^{H} \tilde{\mathbf{H}}_{e, k} \mathbf{w}_{i}\right|^{2}+\left|\left(\hat{\boldsymbol{\theta}}^{q}\right)^{H} \tilde{\mathbf{H}}_{e, k} \mathbf{v}\right|^{2}\right)-\ln \left(1+z_{k}\right),
$$

where $z_{k}=\sum_{i=1}^{K}\left|\left(\hat{\boldsymbol{\theta}}^{q}\right)^{H} \tilde{\mathbf{H}}_{e, k} \mathbf{w}_{i}\right|^{2}+\left|\left(\hat{\boldsymbol{\theta}}^{q}\right)^{H} \tilde{\mathbf{H}}_{e, k} \mathbf{v}\right|^{2}$. The first term in (7) can be rewritten as

$$
\ln \left(1+\sum_{i=1, i \neq k}^{K}\left|\left(\hat{\boldsymbol{\theta}}^{q}\right)^{H} \tilde{\mathbf{H}}_{e, k} \mathbf{w}_{i}\right|^{2}+\left|\left(\hat{\boldsymbol{\theta}}^{q}\right)^{H} \tilde{\mathbf{H}}_{e, k} \mathbf{v}\right|^{2}\right)=\ln \left(1+\boldsymbol{\omega}_{k}^{H} \boldsymbol{\Omega}_{k} \boldsymbol{\Omega}_{k}^{H} \boldsymbol{\omega}_{k}\right)
$$


where $\boldsymbol{\omega}_{k}$ is obtained by removing the $k$-th block from the vector $\left[\mathbf{w}_{1}, \ldots, \mathbf{w}_{K}, \mathbf{v}\right]$ and

$$
\boldsymbol{\Omega}_{k} \boldsymbol{\Omega}_{k}^{H}=\left[\begin{array}{ccc}
\tilde{\mathbf{H}}_{e, k}^{H} \hat{\boldsymbol{\theta}}^{q}\left(\hat{\boldsymbol{\theta}}^{q}\right)^{H} \tilde{\mathbf{H}}_{e, k} & \mathbf{0} & \mathbf{0} \\
\mathbf{0} & \ddots & \mathbf{0} \\
\mathbf{0} & \mathbf{0} & \tilde{\mathbf{H}}_{e, k}^{H} \hat{\boldsymbol{\theta}}^{q}\left(\hat{\boldsymbol{\theta}}^{q}\right)^{H} \tilde{\mathbf{H}}_{e, k}
\end{array}\right] .
$$

Following the matrix inversion lemma $(\mathbf{A}+\mathbf{U B V})^{-1}=\mathbf{A}^{-1}-\mathbf{A}^{-1} \mathbf{U B}\left(\mathbf{I}+\mathbf{V A}^{-1} \mathbf{U B}\right)^{-1} \mathbf{V A}^{-1}$, we obtain $\left(1+\boldsymbol{\omega}_{k}^{H} \boldsymbol{\Omega}_{k}^{H} \boldsymbol{\Omega}_{k} \boldsymbol{\omega}_{k}\right)^{-1}=1-\boldsymbol{\omega}_{k}^{H} \boldsymbol{\Omega}_{k}^{H}\left(\mathbf{I}+\boldsymbol{\Omega}_{k} \boldsymbol{\omega}_{k} \boldsymbol{\omega}_{k}^{H} \boldsymbol{\Omega}_{k}^{H}\right)^{-1} \boldsymbol{\Omega}_{k} \boldsymbol{\omega}_{k}$. For simplicity, we define $\mathbf{Q}_{k}=\mathbf{I}+\boldsymbol{\Omega}_{k} \boldsymbol{\omega}_{k} \boldsymbol{\omega}_{k}^{H} \boldsymbol{\Omega}_{k}^{H}$ to obtain

$$
\ln \left(1+\sum_{i=1, i \neq k}^{K}\left|\left(\hat{\boldsymbol{\theta}}^{q}\right)^{H} \tilde{\mathbf{H}}_{e, k} \mathbf{w}_{i}\right|^{2}+\left|\left(\hat{\boldsymbol{\theta}}^{q}\right)^{H} \tilde{\mathbf{H}}_{e, k} \mathbf{v}\right|^{2}\right)=-\ln \left(1-\boldsymbol{\omega}_{k}^{H} \boldsymbol{\Omega}_{k}^{H} \mathbf{Q}_{k}^{-1} \boldsymbol{\Omega}_{k} \boldsymbol{\omega}_{k}\right) .
$$

Then, we turn (10) into a convex approximation. Based on the concavity of logarithm function $\ln (\gamma) \leq \ln \left(\gamma_{0}\right)+\frac{1}{\gamma_{0}}\left(\gamma-\gamma_{0}\right)$, we have the following relationship

$$
\begin{aligned}
& \ln \left(1+\boldsymbol{\omega}_{k}^{H} \boldsymbol{\Omega}_{k} \boldsymbol{\Omega}_{k}^{H} \boldsymbol{\omega}_{k}\right)=-\ln \left(1-\boldsymbol{\omega}_{k}^{H} \boldsymbol{\Omega}_{k}^{H} \mathbf{Q}_{k}^{-1} \boldsymbol{\Omega}_{k} \boldsymbol{\omega}_{k}\right) \\
& \geq-\ln \left(1-\left(\boldsymbol{\omega}_{k}^{q}\right)^{H} \boldsymbol{\Omega}_{k}^{H}\left(\mathbf{Q}_{k}^{q}\right)^{-1} \boldsymbol{\Omega}_{k} \boldsymbol{\omega}_{k}^{q}\right)-\frac{1-\boldsymbol{\omega}_{k}^{H} \boldsymbol{\Omega}_{k}^{H} \mathbf{Q}_{k}^{-1} \boldsymbol{\Omega}_{k} \boldsymbol{\omega}_{k}}{1-\left(\boldsymbol{\omega}_{k}^{q}\right)^{H} \boldsymbol{\Omega}_{k}^{H}\left(\mathbf{Q}_{k}^{q}\right)^{-1} \boldsymbol{\Omega}_{k} \boldsymbol{\omega}_{k}^{q}}+1 \\
& =-\ln \left(1-\left(\boldsymbol{\omega}_{k}^{q}\right)^{H} \boldsymbol{\Omega}_{k}^{H}\left(\mathbf{Q}_{k}^{q}\right)^{-1} \boldsymbol{\Omega}_{k} \boldsymbol{\omega}_{k}^{q}\right)+\frac{\boldsymbol{\omega}_{k}^{H} \boldsymbol{\Omega}_{k}^{H} \mathbf{Q}_{k}^{-1} \boldsymbol{\Omega}_{k} \boldsymbol{\omega}_{k}-t_{k}^{q}}{1-t_{k}^{q}},
\end{aligned}
$$

where $\boldsymbol{\omega}_{k}^{q}$ is obtained by removing the $k$-th block from the vector $\left[\mathbf{w}_{1}^{q}, \ldots, \mathbf{w}_{K}^{q}, \mathbf{v}^{q}\right]$. In addition, $\mathbf{Q}_{k}^{q}=\mathbf{I}+\boldsymbol{\Omega}_{k} \boldsymbol{\omega}_{k}^{q}\left(\boldsymbol{\omega}_{k}^{q}\right)^{H} \boldsymbol{\Omega}_{k}^{H}$, and $t_{k}^{q}=\left(\boldsymbol{\omega}_{k}^{q}\right)^{H} \boldsymbol{\Omega}_{k}^{H}\left(\mathbf{Q}_{k}^{q}\right)^{-1} \boldsymbol{\Omega}_{k} \boldsymbol{\omega}_{k}^{q}$.

Before we proceed, we find the following lemma about matrix fractional function useful.

Lemma 1 [31]: For any semi-definite positive matrices $\mathbf{A} \in \mathbb{C}^{m \times m}, \mathbf{B} \in \mathbb{C}^{n \times n}, \overline{\mathbf{B}} \in \mathbb{C}^{n \times n}$, $\mathbf{C} \in \mathbb{C}^{m \times n}$, and $\overline{\mathbf{C}} \in \mathbb{C}^{m \times n}$, the following equation holds

$$
\begin{aligned}
& \operatorname{Tr}\left(\mathbf{A} \mathbf{C B}{ }^{-1} \mathbf{C}^{H}\right) \geq \operatorname{Tr}\left(\mathbf{A} \overline{\mathbf{C}} \overline{\mathbf{B}}^{-1} \tilde{\mathbf{C}}^{H}\right)-\operatorname{Tr}\left(\mathbf{A} \overline{\mathbf{C}} \overline{\mathbf{B}}^{-1}(\mathbf{B}-\overline{\mathbf{B}}) \overline{\mathbf{B}}^{-1} \overline{\mathbf{C}}^{H}\right) \\
& +\operatorname{Tr}\left(\mathbf{A}(\mathbf{C}-\overline{\mathbf{C}}) \overline{\mathbf{B}}^{-1} \overline{\mathbf{C}}^{H}\right)+\operatorname{Tr}\left(\mathbf{A} \overline{\mathbf{C}} \overline{\mathbf{B}}^{-1}(\mathbf{C}-\overline{\mathbf{C}})^{H}\right) .
\end{aligned}
$$

Based on the above lemma, we attain the following approximation

$$
\begin{gathered}
\boldsymbol{\omega}_{k}^{H} \boldsymbol{\Omega}_{k}^{H} \mathbf{Q}_{k}^{-1} \boldsymbol{\Omega}_{k} \boldsymbol{\omega}_{k} \geq t_{k}^{q}-\left(\boldsymbol{\omega}_{k}^{q}\right)^{H} \boldsymbol{\Omega}_{k}^{H}\left(\mathbf{Q}_{k}^{q}\right)^{-1}\left(\mathbf{Q}_{k}-\mathbf{Q}_{k}^{q}\right)\left(\mathbf{Q}_{k}^{q}\right)^{-1} \boldsymbol{\Omega}_{k} \boldsymbol{\omega}_{k}^{q} \\
+\left(\boldsymbol{\omega}_{k}-\boldsymbol{\omega}_{k}^{q}\right)^{H} \boldsymbol{\Omega}_{k}^{H}\left(\mathbf{Q}_{k}^{q}\right)^{-1} \boldsymbol{\Omega}_{k} \boldsymbol{\omega}_{k}^{q}+\left(\boldsymbol{\omega}_{k}^{q}\right)^{H} \boldsymbol{\Omega}_{k}^{H}\left(\mathbf{Q}_{k}^{q}\right)^{-1} \boldsymbol{\Omega}_{k}\left(\boldsymbol{\omega}_{k}-\boldsymbol{\omega}_{k}^{q}\right) .
\end{gathered}
$$

Next, we focus on the second term in (7), which can be lower bounded as

$$
-\ln \left(1+z_{k}\right) \geq-\ln \left(1+z_{k}^{q}\right)-\frac{1+z_{k}}{1+z_{k}^{q}}+1,
$$

where $z_{k}^{q}=\sum_{i=1}^{K}\left|\left(\hat{\boldsymbol{\theta}}^{q}\right)^{H} \tilde{\mathbf{H}}_{e, k} \mathbf{w}_{i}^{q}\right|^{2}+\left|\left(\hat{\boldsymbol{\theta}}^{q}\right)^{H} \tilde{\mathbf{H}}_{e, k} \mathbf{v}^{q}\right|^{2}$. 
Thus, by neglecting the constant terms, we obtain the following problem w.r.t. to $\mathbf{w}_{k}$ and $\mathbf{v}$ :

$$
\begin{aligned}
& \min _{\mathbf{w}_{k}, \mathbf{v}} \sum_{k=1}^{K} \varphi_{k}\left\{-\frac{2 \Re\left\{\mathbf{w}_{k}^{H} \tilde{\mathbf{H}}_{k}^{H} \hat{\boldsymbol{\theta}}^{q}\left(\hat{\boldsymbol{\theta}}^{q}\right)^{H} \tilde{\mathbf{H}}_{k} \mathbf{w}_{k}^{q}\right\}}{y_{k}^{q}}+\frac{\left|x_{k}^{q}\right|^{2}\left(y_{k}+\left|x_{k}\right|^{2}\right)}{y_{k}^{q}\left(y_{k}^{q}+\left|x_{k}^{q}\right|^{2}\right)}+\frac{z_{k}}{1+z_{k}^{q}}\right. \\
& \left.+\frac{\left(\boldsymbol{\omega}_{k}^{q}\right)^{H} \boldsymbol{\Omega}_{k}^{H}\left(\mathbf{Q}_{k}^{q}\right)^{-1} \mathbf{Q}_{k}\left(\mathbf{Q}_{k}^{q}\right)^{-1} \boldsymbol{\Omega}_{k} \boldsymbol{\omega}_{k}^{q}}{1-t_{k}^{q}}-\frac{2 \Re\left\{\left(\boldsymbol{\omega}_{k}^{q}\right)^{H} \boldsymbol{\Omega}_{k}^{H}\left(\mathbf{Q}_{k}^{q}\right)^{-1} \boldsymbol{\Omega}_{k} \boldsymbol{\omega}_{k}\right\}}{1-t_{k}^{q}}\right\}
\end{aligned}
$$

s.t. $(5 b)$.

It should be noted that (14) is convex, which can be effectively solved by the CVX tool [45].

\section{B. Phase Shift Matrix Optimization}

In the following, we handle the optimization of $\hat{\boldsymbol{\theta}}$. In fact, the subproblem w.r.t. $\hat{\boldsymbol{\theta}}$ is hard to solve due to the unit modulus constraint.

Specifically, around the given point $\left\{\mathbf{w}_{k}^{q}, \mathbf{v}^{q}, \hat{\boldsymbol{\theta}}^{q}\right\}$, the approximated subproblem w.r.t. $\hat{\boldsymbol{\theta}}$ is given by

$$
\begin{aligned}
& \max _{\hat{\boldsymbol{\theta}}} \sum_{k=1}^{K} \varphi_{k}\left\{\frac{2 \Re\left\{x_{k}^{q} x_{k}\right\}}{y_{k}^{q}}-\frac{\left|x_{k}^{q}\right|^{2}\left(y_{k}+\left|x_{k}\right|^{2}\right)}{y_{k}^{q}\left(y_{k}^{q}+\left|x_{k}^{q}\right|^{2}\right)}-\frac{z_{k}}{1+z_{k}^{q}}\right. \\
& \left.+\ln \left(1+\sum_{i=1, i \neq k}^{K}\left|\hat{\boldsymbol{\theta}}^{H} \tilde{\mathbf{H}}_{e, k} \mathbf{w}_{i}^{q}\right|^{2}+\left|\hat{\boldsymbol{\theta}}^{H} \tilde{\mathbf{H}}_{e, k} \mathbf{v}^{q}\right|^{2}\right)+\mathrm{C}\right\}
\end{aligned}
$$

s.t. $(5 c)$,

where $x_{k}^{q}, y_{k}^{q}$, and $z_{k}^{q}$ has been defined in the previous subsection. In addition, $x_{k}=\hat{\boldsymbol{\theta}}^{H} \tilde{\mathbf{H}}_{k} \mathbf{w}_{k}^{q}$, $y_{k}=\sum_{i=1, i \neq k}^{K}\left|\hat{\boldsymbol{\theta}}^{H} \tilde{\mathbf{H}}_{k} \mathbf{w}_{i}^{q}\right|^{2}+\left|\hat{\boldsymbol{\theta}}^{H} \tilde{\mathbf{H}}_{k} \mathbf{v}^{q}\right|^{2}+1, z_{k}=\sum_{i=1}^{K}\left|\hat{\boldsymbol{\theta}}^{H} \tilde{\mathbf{H}}_{e, k} \mathbf{w}_{i}^{q}\right|^{2}+\left|\hat{\boldsymbol{\theta}}^{H} \tilde{\mathbf{H}}_{e, k} \mathbf{v}^{q}\right|^{2}$, respectively, and $\mathrm{C}=\ln \left(1+\frac{\left|x_{k}^{q}\right|^{2}}{y_{k}^{q}}\right)-\frac{\left|x_{k}^{q}\right|^{2}}{y_{k}^{q}}-\ln \left(1+z_{k}^{q}\right)+\frac{z_{k}^{q}}{1+z_{k}^{q}}$.

The most difficult part in (15a) is the term $\ln \left(1+\sum_{i=1, i \neq k}^{K}\left|\hat{\boldsymbol{\theta}}^{H} \tilde{\mathbf{H}}_{e, k} \mathbf{w}_{i}^{q}\right|^{2}+\left|\hat{\boldsymbol{\theta}}^{H} \tilde{\mathbf{H}}_{e, k} \mathbf{v}^{q}\right|^{2}\right)$. In the following, we will approximate this term via the matrix inversion lemma and the matrix fractional functions.

Specifically, we have $1+\sum_{i=1, i \neq k}^{K}\left|\hat{\boldsymbol{\theta}}^{H} \tilde{\mathbf{H}}_{e, k} \mathbf{w}_{i}^{q}\right|^{2}+\left|\hat{\boldsymbol{\theta}}^{H} \tilde{\mathbf{H}}_{e, k} \mathbf{v}^{q}\right|^{2}=1+\hat{\boldsymbol{\theta}}^{H} \tilde{\mathbf{H}}_{e, k} \mathbf{\Psi}_{k} \mathbf{\Psi}_{k}^{H} \tilde{\mathbf{H}}_{e, k}^{H} \hat{\boldsymbol{\theta}}$, 
where $\boldsymbol{\Psi}_{k} \boldsymbol{\Psi}_{k}^{H}=\sum_{i=1, i \neq k}^{K} \mathbf{w}_{i}^{q}\left(\mathbf{w}_{i}^{q}\right)^{H}+\mathbf{v}^{q}\left(\mathbf{v}^{q}\right)^{H}$. From the matrix inversion lemma, we have

$$
\left(1+\hat{\boldsymbol{\theta}}^{H} \tilde{\mathbf{H}}_{e, k} \boldsymbol{\Psi}_{k} \boldsymbol{\Psi}_{k}^{H} \tilde{\mathbf{H}}_{e, k}^{H} \hat{\boldsymbol{\theta}}\right)^{-1}=1-\hat{\boldsymbol{\theta}}^{H} \tilde{\mathbf{H}}_{e, k} \boldsymbol{\Psi}_{k} \mathbf{P}_{k}^{-1} \boldsymbol{\Psi}_{k}^{H} \tilde{\mathbf{H}}_{e, k}^{H} \hat{\boldsymbol{\theta}}
$$

where $\mathbf{P}_{k}=\mathbf{I}+\boldsymbol{\Psi}_{k}^{H} \tilde{\mathbf{H}}_{e, k}^{H} \hat{\boldsymbol{\theta}} \hat{\boldsymbol{\theta}}^{H} \tilde{\mathbf{H}}_{e, k} \boldsymbol{\Psi}_{k}$. Then, we obtain

$$
\begin{aligned}
& \ln \left(1+\hat{\boldsymbol{\theta}}^{H} \tilde{\mathbf{H}}_{e, k} \boldsymbol{\Psi}_{k} \boldsymbol{\Psi}_{k}^{H} \tilde{\mathbf{H}}_{e, k}^{H} \hat{\boldsymbol{\theta}}\right)=-\ln \left(1-\hat{\boldsymbol{\theta}}^{H} \tilde{\mathbf{H}}_{e, k} \boldsymbol{\Psi}_{k} \mathbf{P}_{k}^{-1} \boldsymbol{\Psi}_{k}^{H} \tilde{\mathbf{H}}_{e, k}^{H} \hat{\boldsymbol{\theta}}\right) \\
& \geq-\ln \left(1-\left(\hat{\boldsymbol{\theta}}^{q}\right)^{H} \tilde{\mathbf{H}}_{e, k} \boldsymbol{\Psi}_{k}\left(\mathbf{P}_{k}^{q}\right)^{-1} \boldsymbol{\Psi}_{k}^{H} \tilde{\mathbf{H}}_{e, k}^{H} \hat{\boldsymbol{\theta}}^{q}\right)+\frac{\hat{\boldsymbol{\theta}}^{H} \tilde{\mathbf{H}}_{e, k} \mathbf{\Psi}_{k} \mathbf{P}_{k}^{-1} \boldsymbol{\Psi}_{k}^{H} \tilde{\mathbf{H}}_{e, k}^{H} \hat{\boldsymbol{\theta}}-u_{k}^{q}}{1-u_{k}^{q}},
\end{aligned}
$$

where $\mathbf{P}_{k}^{q}=\mathbf{I}+\boldsymbol{\Psi}_{k}^{H} \tilde{\mathbf{H}}_{e, k}^{H} \hat{\boldsymbol{\theta}}^{q}\left(\hat{\boldsymbol{\theta}}^{q}\right)^{H} \tilde{\mathbf{H}}_{e, k} \boldsymbol{\Psi}_{k}$, and $u_{k}^{q}=\left(\hat{\boldsymbol{\theta}}^{q}\right)^{H} \tilde{\mathbf{H}}_{e, k} \boldsymbol{\Psi}_{k}\left(\mathbf{P}_{k}^{q}\right)^{-1} \boldsymbol{\Psi}_{k}^{H} \tilde{\mathbf{H}}_{e, k}^{H} \hat{\boldsymbol{\theta}}^{q}$.

Again, using Lemma 1, we obtain the following relationship

$$
\begin{aligned}
& \hat{\boldsymbol{\theta}}^{H} \tilde{\mathbf{H}}_{e, k} \boldsymbol{\Psi}_{k} \mathbf{P}_{k}^{-1} \mathbf{\Psi}_{k}^{H} \tilde{\mathbf{H}}_{e, k}^{H} \hat{\boldsymbol{\theta}} \geq u_{k}^{q}-\left(\hat{\boldsymbol{\theta}}^{q}\right)^{H} \tilde{\mathbf{H}}_{e, k} \Psi_{k}\left(\mathbf{P}_{k}^{q}\right)^{-1}\left(\mathbf{P}_{k}-\mathbf{P}_{k}^{q}\right)\left(\mathbf{P}_{k}^{q}\right)^{-1} \mathbf{\Psi}_{k}^{H} \tilde{\mathbf{H}}_{e, k}^{H} \hat{\boldsymbol{\theta}}^{q} \\
& +\left(\hat{\boldsymbol{\theta}}-\hat{\boldsymbol{\theta}}^{q}\right)^{H} \tilde{\mathbf{H}}_{e, k} \boldsymbol{\Psi}_{k}\left(\mathbf{P}_{k}^{q}\right)^{-1} \boldsymbol{\Psi}_{k}^{H} \tilde{\mathbf{H}}_{e, k}^{H} \hat{\boldsymbol{\theta}}^{q}+\left(\hat{\boldsymbol{\theta}}^{q}\right)^{H} \tilde{\mathbf{H}}_{e, k} \boldsymbol{\Psi}_{k}\left(\mathbf{P}_{k}^{q}\right)^{-1} \mathbf{\Psi}_{k}^{H} \tilde{\mathbf{H}}_{e, k}^{H}\left(\hat{\boldsymbol{\theta}}-\hat{\boldsymbol{\theta}}^{q}\right) .
\end{aligned}
$$

Combining these relationship, we obtain the following problem w.r.t. $\hat{\boldsymbol{\theta}}$ :

$$
\begin{aligned}
& \max _{\hat{\boldsymbol{\theta}}} \sum_{k=1}^{K} \varphi_{k}\left\{\frac{2 \Re\left\{\hat{\boldsymbol{\theta}}^{H} \tilde{\mathbf{H}}_{k} \mathbf{w}_{k}^{q}\left(\mathbf{w}_{k}^{q}\right)^{H} \tilde{\mathbf{H}}_{k}^{H} \hat{\boldsymbol{\theta}}^{q}\right\}}{y_{k}^{q}}+\frac{2 \Re\left\{\hat{\boldsymbol{\theta}}^{H} \tilde{\mathbf{H}}_{e, k} \mathbf{\Psi}_{k}\left(\mathbf{P}_{k}^{q}\right)^{-1} \mathbf{\Psi}_{k}^{H} \tilde{\mathbf{H}}_{e, k}^{H} \hat{\boldsymbol{\theta}}^{q}\right\}}{1-u_{k}^{q}}\right. \\
& \left.-\frac{\left(\hat{\boldsymbol{\theta}}^{q}\right)^{H} \tilde{\mathbf{H}}_{e, k} \mathbf{\Psi}_{k}\left(\mathbf{P}_{k}^{q}\right)^{-1} \mathbf{P}_{k}\left(\mathbf{P}_{k}^{q}\right)^{-1} \mathbf{\Psi}_{k}^{H} \tilde{\mathbf{H}}_{e, k}^{H} \hat{\boldsymbol{\theta}}^{q}}{1-u_{k}^{q}}-\frac{\left|x_{k}^{q}\right|^{2}\left(y_{k}+\left|x_{k}\right|^{2}\right)}{y_{k}^{q}\left(y_{k}^{q}+\left|x_{k}^{q}\right|^{2}\right)}-\frac{z_{k}}{1+z_{k}^{q}}\right\}
\end{aligned}
$$

s.t. $(5 c)$.

For simplicity, we denote $\boldsymbol{\Xi}=\sum_{k=1}^{K} \mathbf{w}_{i}^{q}\left(\mathbf{w}_{i}^{q}\right)^{H}+\mathbf{v}^{q}\left(\mathbf{v}^{q}\right)^{H}$, then we have $\left|x_{k}\right|^{2}+y_{k}=\hat{\boldsymbol{\theta}}^{H} \tilde{\mathbf{H}}_{k} \boldsymbol{\Xi} \tilde{\mathbf{H}}_{k}^{H} \hat{\boldsymbol{\theta}}+$ 1 , and $z_{k}=\hat{\boldsymbol{\theta}}^{H} \tilde{\mathbf{H}}_{e, k} \boldsymbol{\Xi} \tilde{\mathbf{H}}_{e, k}^{H} \hat{\boldsymbol{\theta}}$. Now, by substituting $\mathbf{P}_{k}=\mathbf{I}+\boldsymbol{\Psi}_{k}^{H} \tilde{\mathbf{H}}_{e, k}^{H} \hat{\boldsymbol{\theta}} \hat{\boldsymbol{\theta}}^{H} \tilde{\mathbf{H}}_{e, k} \boldsymbol{\Psi}_{k}$ into the second term in (19a) and neglecting the constant term, each term inside the bracket can be rewritten in the form of $-\hat{\boldsymbol{\theta}}^{H} \mathbf{A}_{k} \hat{\boldsymbol{\theta}}+2 \Re\left\{\hat{\boldsymbol{\theta}}^{H} \mathbf{b}_{k}\right\}$, where $\mathbf{A}_{k}$ and $\mathbf{B}_{k}$ are, respectively, given by $\mathbf{A}_{k}=\frac{\tilde{\mathbf{H}}_{e, k} \boldsymbol{\Psi}_{k}\left(\mathbf{P}_{k}^{q}\right)^{-1} \boldsymbol{\Psi}_{k}^{H} \tilde{\mathbf{H}}_{e, k}^{H} \hat{\boldsymbol{\theta}}^{q}\left(\hat{\boldsymbol{\theta}}^{q}\right)^{H} \tilde{\mathbf{H}}_{e, k} \boldsymbol{\Psi}_{k}\left(\mathbf{P}_{k}^{q}\right)^{-1} \boldsymbol{\Psi}_{k}^{H} \tilde{\mathbf{H}}_{e, k}^{H}}{1-u_{k}^{q}}+\frac{\tilde{\mathbf{H}}_{e, k} \boldsymbol{\Xi} \tilde{\mathbf{H}}_{e, k}^{H}}{1+z_{k}^{q}}+\frac{\left|x_{k}^{q}\right|^{2} \tilde{\mathbf{H}}_{k} \boldsymbol{\Xi} \tilde{\mathbf{H}}_{k}^{H}}{y_{k}^{q}\left(y_{k}^{q}+\left|x_{k}^{q}\right|^{2}\right)}$,

$\mathbf{b}_{k}=\frac{\tilde{\mathbf{H}}_{k} \mathbf{w}_{k}^{q}\left(\mathbf{w}_{k}^{q}\right)^{H} \tilde{\mathbf{H}}_{k}^{H} \hat{\boldsymbol{\theta}}^{q}}{y_{k}^{q}}+\frac{\tilde{\mathbf{H}}_{e, k} \boldsymbol{\Psi}_{k}\left(\mathbf{P}_{k}^{q}\right)^{-1} \Psi_{k}^{H} \tilde{\mathbf{H}}_{e, k}^{H} \hat{\boldsymbol{\theta}}^{q}}{1-u_{k}^{q}}$ 
Combining these relationships, we obtain the following subproblem w.r.t. to $\hat{\boldsymbol{\theta}}$ :

$$
\begin{aligned}
& \min _{\hat{\boldsymbol{\theta}}} \hat{\boldsymbol{\theta}}^{H} \mathbf{A} \hat{\boldsymbol{\theta}}-2 \Re\left\{\hat{\boldsymbol{\theta}}^{H} \mathbf{b}\right\} \\
& \text { s.t. }(5 \mathrm{c}),
\end{aligned}
$$

where $\mathbf{A}=\sum_{k=1}^{K} \varphi_{k} \mathbf{A}_{k}$ and $\mathbf{b}=\sum_{k=1}^{K} \varphi_{k} \mathbf{b}_{k}$

The challenge in (21) is the unit modulus constraint (21b). In this work, we propose two methods to tackle this obstacle. Firstly, we investigate the ADMM method.

1) ADMM Method: To fit (21) into the ADMM framework, we introduce a slack variable $\boldsymbol{r} \in \mathbb{C}^{M+1}$ and rewrite $(21)$ as

$$
\begin{array}{ll}
\min _{\boldsymbol{r}, \hat{\boldsymbol{\theta}}} & \boldsymbol{r}^{H} \mathbf{A} \boldsymbol{r}-2 \Re\left\{\boldsymbol{r}^{H} \mathbf{b}\right\} \\
\text { s.t. } & \left|\hat{\theta}_{m}\right|=1, \forall m \in \mathcal{M}, \hat{\theta}_{M+1}=1, \\
& \quad \boldsymbol{r}=\hat{\boldsymbol{\theta}} .
\end{array}
$$

The augmented Lagrange of (22) is

$$
\mathcal{L}(\boldsymbol{r}, \hat{\boldsymbol{\theta}}, \boldsymbol{p})=\boldsymbol{r}^{H} \mathbf{A} \boldsymbol{r}-2 \Re\left\{\boldsymbol{r}^{H} \mathbf{b}\right\}-\Re\left\{\boldsymbol{p}^{H}(\boldsymbol{r}-\hat{\boldsymbol{\theta}})\right\}+\frac{\rho}{2}\|\boldsymbol{r}-\hat{\boldsymbol{\theta}}\|^{2},
$$

where $\rho \geq 0, \boldsymbol{p} \in \mathbb{C}^{M+1}$ is the Lagrange multiplier associated with the (22c).

Let $\left(\boldsymbol{r}^{0}, \hat{\boldsymbol{\theta}}^{0}, \boldsymbol{p}^{0}\right)$ be the initial variables. The ADMM method cyclically carries out the following three steps:

$$
\begin{aligned}
& \boldsymbol{r}^{n+1}=\arg \min _{\boldsymbol{r}} \mathcal{L}\left(\boldsymbol{r}^{n}, \hat{\boldsymbol{\theta}}^{n}, \boldsymbol{p}^{n}\right), \\
& \hat{\boldsymbol{\theta}}^{n+1}=\arg \min _{\left|\hat{\theta}_{m}\right|=1, \forall m \in \mathcal{M}} \mathcal{L}\left(\boldsymbol{r}^{n+1}, \hat{\boldsymbol{\theta}}^{n}, \boldsymbol{p}^{n}\right), \\
& \boldsymbol{p}^{n+1}=\boldsymbol{p}^{n}-\rho\left(\boldsymbol{r}^{n+1}-\hat{\boldsymbol{\theta}}^{n+1}\right),
\end{aligned}
$$

for $n=1, \ldots$ until certain stopping criterion is met.

The merit of the ADMM method is that each subproblem has a closed form solution. Using the first-order optimization condition, we obtain the following solution for (24a)

$$
2 \mathbf{A} \boldsymbol{r}^{n+1}-2 \mathbf{b}-\boldsymbol{p}^{n}-\rho\left(\hat{\boldsymbol{\theta}}^{n}-\boldsymbol{r}^{n+1}\right)=\mathbf{0}
$$


which can be rearranged as

$$
\boldsymbol{r}^{n+1}=(\rho \mathbf{I}+2 \mathbf{A})^{-1}\left(2 \mathbf{b}+\rho \hat{\boldsymbol{\theta}}^{n}+\boldsymbol{p}^{n}\right) .
$$

Next, (24b) is equivalent to $\min _{\left|\hat{\theta}_{m}\right|=1, \forall m \in \mathcal{M}}\left\|\hat{\boldsymbol{\theta}}-\left(\boldsymbol{r}^{n+1}-\rho^{-1} \boldsymbol{p}^{n}\right)\right\|^{2}$, which has a closed-form solution

$$
\left[\hat{\boldsymbol{\theta}}^{n+1}\right]_{m}= \begin{cases}\frac{\left[\boldsymbol{r}^{n+1}-\rho^{-1} \boldsymbol{p}^{n}\right]_{m}}{\left|\left[\boldsymbol{r}^{n+1}-\rho^{-1} \boldsymbol{p}^{n}\right]_{m}\right|}, & \text { if }\left[\boldsymbol{r}^{n+1}-\rho^{-1} \boldsymbol{p}^{n}\right]_{m} \neq 0 \\ {\left[\hat{\boldsymbol{\theta}}^{n}\right]_{m},} & \text { otherwise, }\end{cases}
$$

where $[\hat{\boldsymbol{\theta}}]_{m}$ denotes the $m$-th entry of $\hat{\boldsymbol{\theta}}$. Lastly, from $(24 \mathrm{c})$ and (25), we obtain that $\boldsymbol{p}^{n+1}=$ $2 \mathrm{~A} \boldsymbol{r}^{n+1}-2 \mathbf{b}$. For the convergence of the ADMM algorithm, we have the following Lemma.

Lemma 2 [41]: No matter whether $\hat{\boldsymbol{\theta}}$ belongs to the continuous or the discrete set, the ADMM algorithm guarantees to converge, when the penalty parameter $\rho$ satisfies: $\rho \mathbf{I} / 2-\mathbf{A} \succ \mathbf{0}$.

The ADMM algorithm is summarized in Algorithm 1.

Algorithm 1 The ADMM Algorithm for Problem (22).

1: Initialization: $n=0$, set a feasible point $\left\{\boldsymbol{r}^{0}, \hat{\boldsymbol{\theta}}^{0}, \boldsymbol{p}^{0}\right\}$, set the accuracy $\kappa$, and the penalty factor $\rho=\ell\|\mathbf{A}\|$, where $\ell$ is the minimum integer which satisfies $\rho \mathbf{I} / 2-\mathbf{A} \succ \mathbf{0}$.

2: repeat

a) Calculate $\boldsymbol{r}^{n+1}$ via (26).

b) Calculate $\hat{\boldsymbol{\theta}}^{n+1}$ via (27).

c) Calculate $\boldsymbol{p}^{n+1}=2 \mathbf{A} \boldsymbol{r}^{n+1}-2 \mathbf{b}$.

d) $n \leftarrow n+1$.

3: until $R_{s}^{n}-R_{s}^{n-1}<\kappa$.

4: Output $\left\{\boldsymbol{r}^{\star}, \hat{\boldsymbol{\theta}}^{\star}, \boldsymbol{p}^{\star}\right\}$.

2) EBCD Method: In this part, we propose the EBCD method to solve (21).

Let us denote the $(i, j)$-th entry of $\mathbf{A}$ as $a_{i, j}$, and the $i$-th entry of $\mathbf{b}$ as $b_{i}$. Then, $\hat{\boldsymbol{\theta}}^{H} \mathbf{A} \hat{\boldsymbol{\theta}}$ can be expressed as

$$
\hat{\boldsymbol{\theta}}^{H} \mathbf{A} \hat{\boldsymbol{\theta}}=\sum_{i=1}^{M+1} \sum_{j=1}^{M+1} \hat{\theta}_{i}^{\dagger} a_{i, j} \hat{\theta}_{j}=\hat{\theta}_{m}^{\dagger} a_{m, m} \hat{\theta}_{m}+2 \Re\left\{\sum_{j=1, j \neq m}^{M+1} \hat{\theta}_{m}^{\dagger} a_{m, j} \hat{\theta}_{j}\right\}+\sum_{\substack{i=1, i \neq m \\ i \neq m}}^{M+1} \sum_{\substack{j=m \\ j \neq m}}^{M+1} \hat{\theta}_{i}^{\dagger} a_{i, j} \hat{\theta}_{j},
$$

where we exploit the feature that $a_{i, j}=a_{j, i}^{\dagger}$, since $\mathbf{A}$ is a hermitian matrix. Similarly, $\hat{\boldsymbol{\theta}}^{H} \mathbf{b}$ can 
be expressed as

$$
\hat{\boldsymbol{\theta}}^{H} \mathbf{b}=\sum_{i=1}^{M+1} \hat{\theta}_{i}^{\dagger} b_{i}=\hat{\theta}_{m}^{\dagger} b_{m}+\sum_{i=1, i \neq m}^{M+1} \hat{\theta}_{i}^{\dagger} b_{i} .
$$

By substituting (28) and (29) into (21a) and dropping all the irrelevant constant terms, we obtain the following problem w.r.t. $\hat{\theta}_{m}$, given all the other $\hat{\theta}_{i}(i \neq m)$ fixed:

$$
\begin{aligned}
& \min _{\hat{\theta}_{m}} \hat{\theta}_{m}^{\dagger} a_{m, m} \hat{\theta}_{m}-2 \Re\left\{\hat{\theta}_{m}^{\dagger} b_{m}-\sum_{j=1, j \neq m}^{M+1} \hat{\theta}_{m}^{\dagger} a_{m, j} \hat{\theta}_{j}\right\} \\
& \text { s.t. }\left|\hat{\theta}_{m}\right|=1, \forall m \in \mathcal{M}, \hat{\theta}_{M+1}=1 .
\end{aligned}
$$

Since $\left|\hat{\theta}_{m}\right|^{2}=1$, (30a) can be simplified as $a_{m, m}-2 \Re\left\{\hat{\theta}_{m}^{\dagger} \tilde{b}_{m}\right\}$, where $\tilde{b}_{m}=b_{m}-\sum_{j=1, j \neq m}^{M+1} a_{m, j} \hat{\theta}_{j}$. Then, the optimal $\hat{\theta}_{m}^{\star}$ for (30) is given by $\hat{\theta}_{m}^{\star}=\tilde{b}_{m} /\left|\tilde{b}_{m}\right|$.

Finally, all the RC can be optimized based on in the order from $m=1$ to $m=M$ and repeatedly until convergence [41].

\section{Overall Algorithm and Convergence Analysis}

At last, we have turned (5) into a solvable problem, where the two subproblems can be solved with the respective methods. The overall procedure is given in Algorithm 2, where $R_{s}^{q}$ denotes the obtained WSSR in the $q$-th iteration, and $\kappa$ denotes the stopping threshold.

Algorithm 2 The AO Algorithm for Problem (5).

1: Initialization: $q=1$, set $P_{s}, N_{t}, M, \sigma_{b}, \sigma_{e}, \mathbf{h}_{d, k}, \mathbf{h}_{r, k}, \mathbf{h}_{d, e, k}, \mathbf{h}_{r, e, k}$, and $\kappa$.

2: repeat

a) Obtain $\left\{\mathbf{w}_{k}^{q}, \mathbf{v}^{q}\right\}$ via solving (14) using $\mathrm{CVX}$, with fixed $\left\{\mathbf{w}_{k}^{q-1}, \mathbf{v}^{q-1}, \hat{\boldsymbol{\theta}}^{q-1}\right\}$.

b) Obtain $\hat{\boldsymbol{\theta}}^{q}$ via solving (21) using the ADMM or EBCD method, with fixed $\left\{\mathbf{w}_{k}^{q}, \mathbf{v}^{q}, \hat{\boldsymbol{\theta}}^{q-1}\right\}$.

c) Update the fixed point $\left\{\mathbf{w}_{k}^{q-1}, \mathbf{v}^{q-1}, \hat{\boldsymbol{\theta}}^{q-1}\right\}$ using the obtained point $\left\{\mathbf{w}_{k}^{q}, \mathbf{v}^{q}, \hat{\boldsymbol{\theta}}^{q}\right\}$.

d) $q \leftarrow q+1$.

3: until $R_{s}^{q}-R_{s}^{q-1}<\kappa$.

4: Output $\left(\mathbf{w}_{k}^{\star}, \mathbf{v}^{\star}, \hat{\boldsymbol{\theta}}^{\star}\right)$. 
For the convergence of the $\mathrm{AO}$ algorithm, we have the following two Theorems.

Theorem 1: The value of the objective function increases in each iteration of Algorithm 1, i.e., $R_{s}\left(\left\{\mathbf{w}_{k}^{q}\right\}_{k=1}^{K}, \mathbf{v}^{q}, \hat{\boldsymbol{\theta}}^{q}\right) \leq R_{s}\left(\left\{\mathbf{w}_{k}^{q+1}\right\}_{k=1}^{K}, \mathbf{v}^{q+1}, \hat{\boldsymbol{\theta}}^{q+1}\right)$, which guarantees to converge to a locally optimal point.

Proof: Please refer to Appendix A.

Theorem 2: The corresponding solution $\left(\left\{\mathbf{w}_{k}^{\star}\right\}_{k=1}^{K}, \mathbf{v}^{\star}, \hat{\boldsymbol{\theta}}^{\star}\right)$ will converge to a Karush-KuhnTucker (KKT) point finally.

Proof: Please refer to Appendix B.

\section{Complexity Comparisons}

In this subsection, we analyze the complexity of the ADMM and EBCD methods, and compare that with several other methods. Firstly, when utilizing the ADMM method to solve (21), according to [40], the complexity is $\mathcal{O}\left(T_{\mathrm{ADMM}} M^{2}+M^{3}\right)$, with $T_{\mathrm{ADMM}}$ being the total ADMM iteration numbers. On the other hand, when utilizing the EBCD method to solve (21), according to [41], the complexity is $\mathcal{O}\left(T_{\mathrm{EBCD}} M^{2}\right)$, with $T_{\mathrm{EBCD}}$ being the total EBCD iteration numbers. The proposed algorithms enjoy polynomial time complexity, which is suitable for practical implementation.

Theoretically, the ADMM method incurs higher computational complexity than the EBCD method. However, instead of the serial operation in the EBCD method, the variables are updated simultaneously in the ADMM method. Hence, the ADMM algorithm may converge faster than the EBCD algorithm [41].

In addition, for the commonly used MM method, based on [14] and [16], the total complexity of the $\mathrm{MM}$ algorithm is given by $\mathcal{C}_{\mathrm{MM}}=\mathcal{O}\left(T_{\mathrm{MM}} M^{2}+M^{3}\right)$, where $T_{\mathrm{MM}}$ denotes the total iteration numbers required by the $\mathrm{MM}$ algorithm. From this result, we can conclude that the ADMM and MM method have similar complexity. 


\section{Extension to Other SCEnARios}

\section{A. Discrete Phase Shifter Case}

In the previous part, we focus on the continuous phase shifter case. In fact, we can apply the obtained $\theta_{m} \in \mathcal{F}_{1}$, into the discrete phase shifter case $\theta_{m} \in \mathcal{F}_{2}$ directly. Specifically, we denote the solution of $\theta_{m} \in \mathcal{F}_{1}$ and $\theta_{m} \in \mathcal{F}_{2}$ as $\theta_{m}^{(1)}$ and $\theta_{m}^{(2)}$, respectively. Then, we project $\theta_{m}^{(1)}$ into $\mathcal{F}_{2}$ to obtain $\theta_{m}^{(2)}$, i.e., $\theta_{m}^{(2)}=e^{j \phi_{q^{\star}}}$, where $q^{\star}=\underset{1 \leq q \leq \tau}{\arg \min }\left|\theta_{m}^{(1)}-e^{j \phi_{q}}\right|$.

It should be noted that the discrete procedure dose not conduct in the end of the AO algorithm. However, during each step of the AO iteration, we discrete the obtained $\mathrm{RC}$ in the continuous case to the discrete set, and then set the obtained discrete RC as a fixed point to update the $\mathrm{BF}$ and $\mathrm{AN}$. The whole procedure continue until converge. Besides, the procedure to update $\left(\left\{\mathbf{w}_{k}\right\}_{k=1}^{K}, \mathbf{v}, \hat{\boldsymbol{\theta}}\right)$ is similar to that in the previous part, and thus is omitted here for brevity. Although Theorems 1 and 2 can not be applied in the discrete RC case directly, according to [41], if the discrete phase shifts $\hat{\boldsymbol{\theta}}^{q}$ satisfies $f\left(\hat{\boldsymbol{\theta}}^{q}\right) \geq f\left(\hat{\boldsymbol{\theta}}^{q-1}\right)$, where $f\left(\hat{\boldsymbol{\theta}}^{q}\right)$ denotes the objective value of (22) or (30) in the $q$-th iteration, then, the proposed AO algorithm is guaranteed to converge in the discrete RC case. In fact, the proposed AO algorithm works well in the discrete RC case, which will be verified in the simulation part.

\section{B. Multiple IRSs Scenario}

Let us assume that the system has $L$ IRSs, each of which has $M_{l}$ reflection elements and the phase shift matrix of the $l$-th IRS is denoted as $\Theta_{l}$. In addition, we denote the channels between the BS and the $l$-th IRS, between the $l$-th IRS and the $k$-th Bob, between the $l$-th IRS and the $k$-th Eve as $\mathbf{G}_{l}, \mathbf{h}_{r, k, l}^{H}$ and $\mathbf{h}_{r, e, k, l}^{H}$, respectively. It should be pointed that since IRSs are passive, their reflected signals decay in power rapidly over distance. Thus, as long as IRSs are deployed sufficiently far apart from each other, their mutual interference is practically negligible [1]. In addition, similar to [32], we assume that the signals reflected by the IRS are typically tilting down to the ground to serve the Bobs. Hence, the inter-surface reflection between each IRS is neglected in the investigated multiple IRSs scenario. 
Then, the received signals at the $k$-th Bob and the $k$-th Eve can be respectively written as

$$
\begin{aligned}
& y_{k}=\left(\mathbf{h}_{d, k}^{H}+\sum_{l=1}^{K} \mathbf{h}_{r, k, l}^{H} \boldsymbol{\Theta}_{l}^{H} \mathbf{G}_{l}\right) \mathbf{x}+n_{k}=\hat{\boldsymbol{\theta}}^{H} \mathbf{H}_{k} \mathbf{x}+n_{k}, \\
& y_{e, k}=\left(\mathbf{h}_{d, k}^{H}+\sum_{l=1}^{K} \mathbf{h}_{r, k, l}^{H} \boldsymbol{\Theta}_{l}^{H} \mathbf{G}_{l}\right) \mathbf{x}+n_{k}=\hat{\boldsymbol{\theta}}^{H} \mathbf{H}_{e, k} \mathbf{x}+n_{e, k} .
\end{aligned}
$$

where $\hat{\boldsymbol{\theta}}=\left[\boldsymbol{\theta}_{1}^{H}, \ldots, \boldsymbol{\theta}_{L}^{H}, 1\right]^{H}, \mathbf{H}_{k}=\left[\operatorname{diag}\left(\mathbf{h}_{r, k, 1}^{H}\right) \mathbf{G}_{1}, \ldots, \operatorname{diag}\left(\mathbf{h}_{r, k, L}^{H}\right) \mathbf{G}_{L}, \mathbf{h}_{d, k}^{H}\right]^{T}$, and $\mathbf{H}_{e, k}=$ $\left[\operatorname{diag}\left(\mathbf{h}_{r, e, k, 1}^{H}\right) \mathbf{G}_{1}, \ldots, \operatorname{diag}\left(\mathbf{h}_{r, e, k, L}^{H}\right) \mathbf{G}_{L}, \mathbf{h}_{d, e, k}^{H}\right]^{T}$, respectively.

Thus, the previous method can be applied to the multiple IRSs scenario directly.

\section{Simulation Results}

In this section, we provide simulation results to assess the performance of the proposed algorithm. The simulation scenario is shown in Fig. 2, where there are one BS, one IRS, 4 BobEve groups. The coordinates of the BS and the IRS are $(0 \mathrm{~m}, 10 \mathrm{~m}),(50 \mathrm{~m}, 20 \mathrm{~m})$, respectively. The antenna array of the BS is parallel to the $y$ axis. In addition, the heights of the BS and IRS are $20 \mathrm{~m}$ and $10 \mathrm{~m}$, respectively, while the heights of all Bobs and Eves are $1.5 \mathrm{~m}$. For the locations of the Bob-Eve groups, we assume that the Bob-Eve groups are scattered in the corners of a square. Specifically, the coordinates for Bobs are $(45 \mathrm{~m}, 5 \mathrm{~m}),(55 \mathrm{~m}, 5 \mathrm{~m}),(45 \mathrm{~m},-5 \mathrm{~m})$, and $(55 \mathrm{~m},-5 \mathrm{~m})$, respectively, while each Eve is randomly placed in a circle centered at the nearest Bob, with radius of $2 \mathrm{~m}$, respectively.

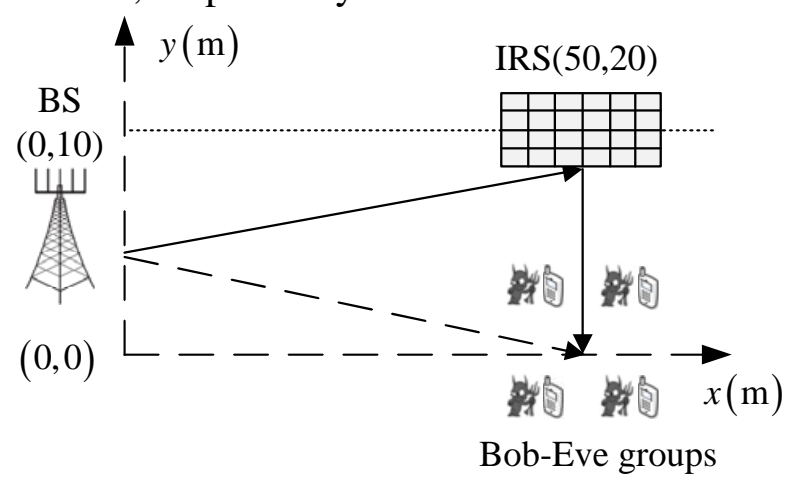

Fig. 2. The IRS-aided communication scenario in the simulation.

Unless otherwise specified, the simulation settings are assumed as follows: $K=4, N=5$, $M=50, \tau=8$, the unit bandwidth is considered and $P_{T}=20 \mathrm{dBm}, \sigma_{k}^{2}=\sigma_{k, e}^{2}=-80 \mathrm{dBm}$, 
$\varphi_{k}=1 / K, \forall k$. The large-scale path loss is given by $\mathrm{PL}=\mathrm{PL}_{0}-10 \alpha \log _{10}\left(\frac{d}{d_{0}}\right)$, where $\mathrm{PL}_{0}$ is the path loss at the reference distance $d_{0}, d$ is the link distance, $\alpha$ is the path loss exponent. In the simulations, we set $\mathrm{PL}_{0}=-30 \mathrm{~dB}$ and $d_{0}=1 \mathrm{~m}$. Due to the extensive obstacles and scatterers, the path loss exponent between the BS and the Bobs/Eves is given by $\alpha_{\mathrm{B}}=4$, and the small-scale fading is assumed to be Rayleigh fading [14]. On the other hand, by carefully choosing the location of the IRS, the IRS-related link can experience nearly free-space path loss. Thus, we set the path loss exponents of the IRS-related links by $\alpha_{\mathrm{I}}=2$ [16]. As for the small-scale fading, take $\mathbf{G}$ as example, which is modeled as $\mathbf{G}=\sqrt{\frac{\beta}{\beta+1}} \mathbf{G}^{\mathrm{LoS}}+$ $\sqrt{\frac{1}{\beta+1}} \mathbf{G}^{\mathrm{NLoS}}$, where $\beta$ is the Rician factor, $\mathbf{G}^{\mathrm{LoS}}$ is the line-of-sight $(\mathrm{LoS})$ component, and $\mathbf{G}^{\mathrm{NLoS}}$ is the non-LoS (NLoS) component that is Rayleigh fading. Besides, $\mathrm{G}^{\mathrm{LoS}}$ is given by $\mathrm{G}^{\mathrm{LoS}}=$ $\mathbf{a}_{D_{\mathrm{r}}}\left(\vartheta^{\mathrm{AoA}}\right) \mathbf{a}_{D_{\mathrm{t}}}^{H}\left(\vartheta^{\mathrm{AoD}}\right)$, where $\mathbf{a}_{D_{\mathrm{r}}}\left(\vartheta^{\mathrm{AoA}}\right)=\left[1, e^{j \frac{2 \pi d_{\mathrm{r}}}{\lambda} \sin \vartheta^{\mathrm{AoA}}}, \cdots, e^{j \frac{2 \pi d_{\mathrm{r}}}{\lambda}\left(D_{\mathrm{r}}-1\right) \sin \vartheta^{\mathrm{AoA}}}\right]^{T}$, and $\mathbf{a}_{D_{\mathrm{t}}}\left(\vartheta^{\mathrm{AoD}}\right)=\left[1, e^{j \frac{2 \pi d_{\mathrm{t}}}{\lambda} \sin \vartheta^{\mathrm{AoD}}}, \cdots, e^{j \frac{2 \pi d_{\mathrm{t}}}{\lambda}\left(D_{\mathrm{t}}-1\right) \sin \vartheta^{\mathrm{AoD}}}\right]^{T}$, respectively, $D_{\mathrm{r}}$ and $D_{\mathrm{t}}$ are the number of antennas at the receiver $(\mathrm{Rx})$ and the transmitter $(\mathrm{Tx})$, respectively, while $d_{\mathrm{r}}$ and $d_{\mathrm{t}}$ are the antenna separation distance at the $\mathrm{Rx}$ and the Tx, respectively, $\lambda$ is the wavelength, $\vartheta^{\mathrm{AoD}}$ and $\vartheta^{\mathrm{AoA}}$ are the angle of departure and arrival, respectively. In the following, similar to [33], we set $\beta=3, d_{\mathrm{r}} / \lambda=d_{\mathrm{t}} / \lambda=0.5, \vartheta^{\mathrm{AoA}}=\tan ^{-1}\left(\frac{y_{\mathrm{IRS}}-y_{\mathrm{BS}}}{x_{\mathrm{IRS}}-x_{\mathrm{BS}}}\right)$, and $\vartheta^{\mathrm{AoD}}=\pi-\vartheta^{\mathrm{AoA}}$, respectively.

\section{A. Convergence Behaviour}

Here, we investigate the convergence behaviour of the proposed $\mathrm{AO}$ algorithm. Both the outer layer iteration and the inner layer iteration are considered. Specifically, the entire AO algorithm is termed as the outer layer iteration, while the procedure of the ADMM or EBCD algorithm is termed as the inner layer iterations. We first investigate the convergence behaviour of the inner layer iterations.

1) Convergence Behaviour of the ADMM or EBCD Method: Fig. 3 shows the convergence performance of the ADMM or EBCD algorithm in the first iteration of the AO algorithm for different phase shift numbers. From Fig. 3, we can see that the WSSR increases with the iteration numbers for the two methods, and gradually converges. Moreover, high secrecy rate can be 


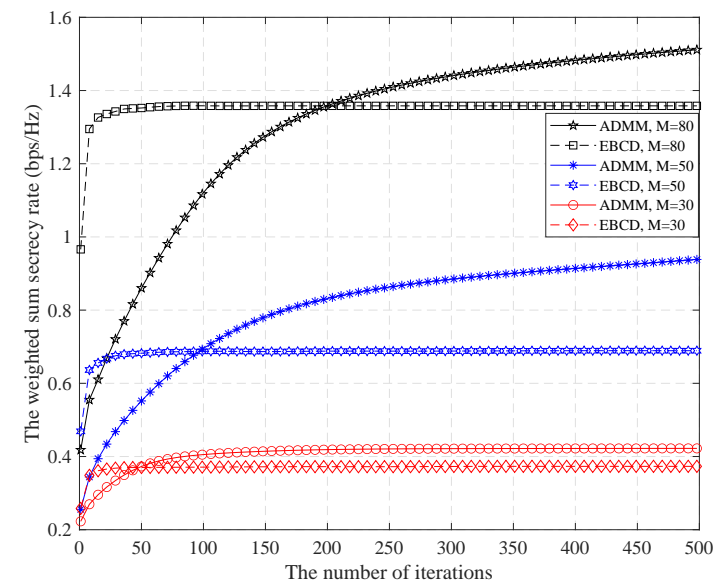

Fig. 3. The inner convergence behaviour of the proposed methods.

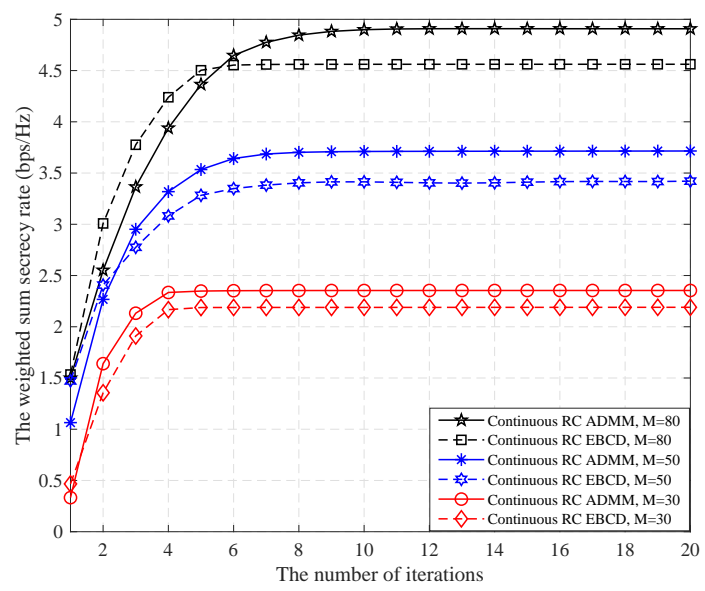

Fig. 4. The outer convergence behaviour of the proposed method.

achieved by using more IRS elements. However, larger $M$ incurs higher complexity, since more variables need to be optimized and lead to slower convergence. Besides, given the same phase shifts numbers and channel condition, the EBCD method tends to converge more quickly than the ADMM method. However, the ADMM method can achieve higher WSSR than the EBCD method.

2) Convergence Behaviour of the AO Method: Now, we investigate the convergence behaviour of the outer AO algorithm. Both the AO-ADMM and the AO-EBCD algorithms are examined. Fig. 4 shows the WSSR versus the number of iterations with different phase shift numbers. Similarly observation to the case of the inner layer iteration can be observed, that larger $M$ leads to more iteration numbers. However, for different values of $M$, both the AO-ADMM and the AO-EBCD algorithms always converge within 20 iterations, which confirms the practicality of the proposed design. Besides, given the same phase shift numbers and channel condition, the AO-ADMM and the AO-EBCD methods have a similar convergence speed, while the AOADMM method can achieve higher WSSR than the AO-EBCD method.

As an important extension, here we examine the convergence performance of the outer AOADMM algorithm in the case of discrete RC. From the results in Fig. 5, we can see that in both the continuous $\mathrm{RC}$ and discrete $\mathrm{RC}$ case, the proposed AO-ADMM method always converges in 


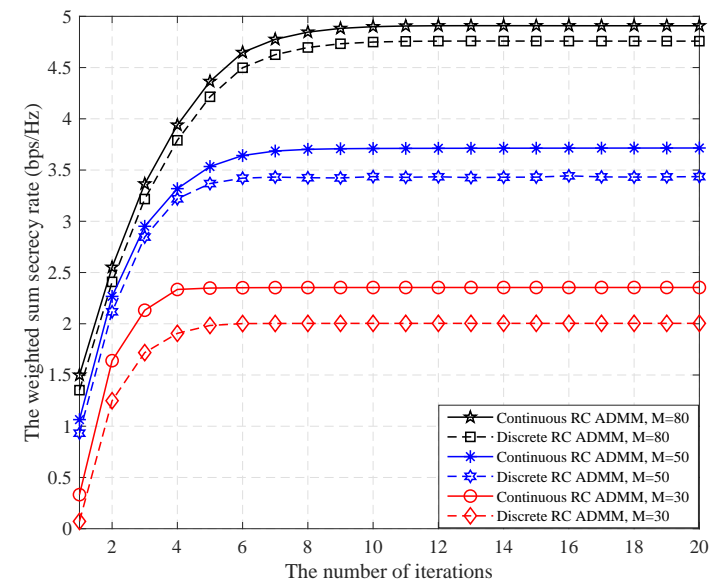

Fig. 5. The outer convergence behaviour of the proposed method in the case of discrete RC.

a few number of iterations. Besides, Fig. 5 shows that given the same phase shift numbers and channel condition, the discrete RC case suffers from some performance loss when compared with the continuous RC. This is mainly due to the quantized error introduced by the limited bits in the discrete RC case.

\section{B. Performance Evaluation}

Here, we evaluate the system performance against several system parameters. Both the continuous and discrete $\mathrm{RC}$ are considered. Besides, we compare the proposed methods with the following schemes: 1) the MM method, which is used to design the phase shifters; 2) with IRS but without AN method; 3) without IRS but with AN method; 4) without IRS and without AN method. These methods are labelled as "Continuous RC ADMM", "Discrete RC ADMM", “Continuous RC EBCD”, “Discrete RC EBCD”, "MM method”, "No-AN method”, "No-IRS method", and "No-IRS No-AN method", respectively.

Firstly, we investigate the WSSR versus the transmit power budget for various schemes. The results are shown in Fig. 6. As we can see, the WSSR obtained by all these schemes increases with the increasing of $P_{T}$. For all the designs which consider IRS, the proposed AO-ADMM method in the continuous RC case obtains slightly better performance than other designs, while 


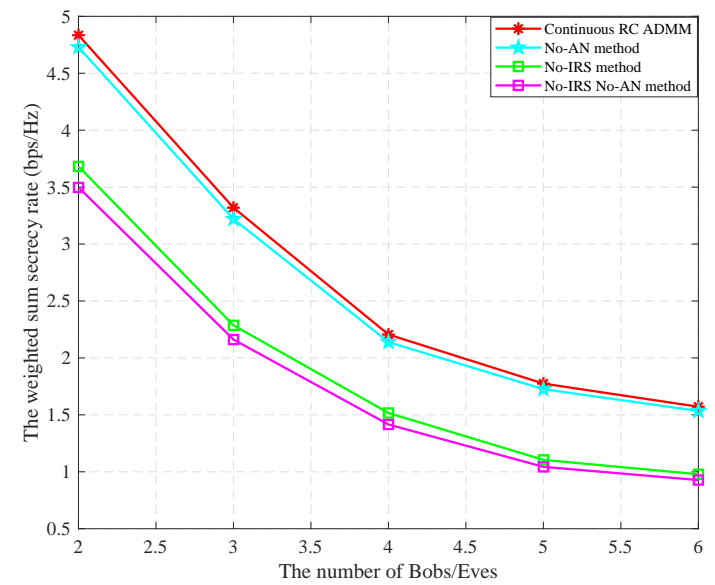

Fig. 7. The WSSR versus the number of Bobs/Eves.

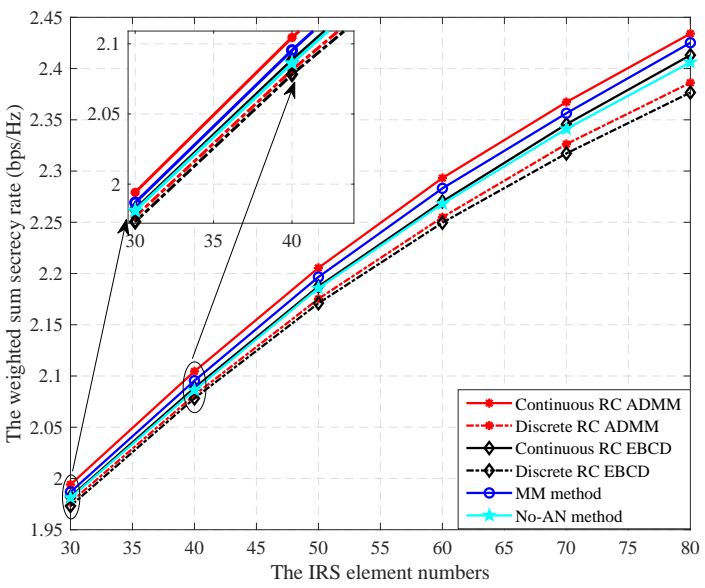

Fig. 8. The WSSR versus the IRS element numbers. the discrete RC case suffers from some performance loss. In addition, all these IRS-aided methods significantly outperform the no-IRS schemes, which demonstrates the merit of utilizing the IRS. Besides, in IRS-aided case, the no-AN method achieves nearly performance than the AN-aided method, however, in the no-IRS-aided scenario, AN can improve the security more evidently.

Then, in Fig. 7, we show the WSSR versus the number of Bobs/Eves $K$. From this figure, we can see that the WSSR obtained by all these schemes decreases with the increasing of $K$. This is mainly due to the fact that with the increasing of $K$, the inter-user interference becomes larger, thus the WSSR tends to decrease. In addition, with the increasing of $K$, the performance gap between the AN-aided schemes and the no-AN-aided methods tends to decrease. This arises from the fact that the inter-user interference play a similar role as $\mathrm{AN}$, thus the effect of $\mathrm{AN}$ in improving the security is limited in multi-user network, especially for large $K$.

Next, in Fig. 8, we show the WSSR versus the IRS element numbers $M$. From this figure, we can see that for all these IRS-aided methods, the WSSR tends to increase with the value of $M$. The performance gain mainly comes from the fact that with larger $M$, more signals can reach the IRS, and the sum of the reflected signals increases, provided that the phase shifts are appropriately optimized. This result shows that a larger IRS can further improve the secrecy performance when optimizing the phase shifts properly.

To further show the effect of IRS in improving the security, in Fig. 9 and Fig. 10, we show the 


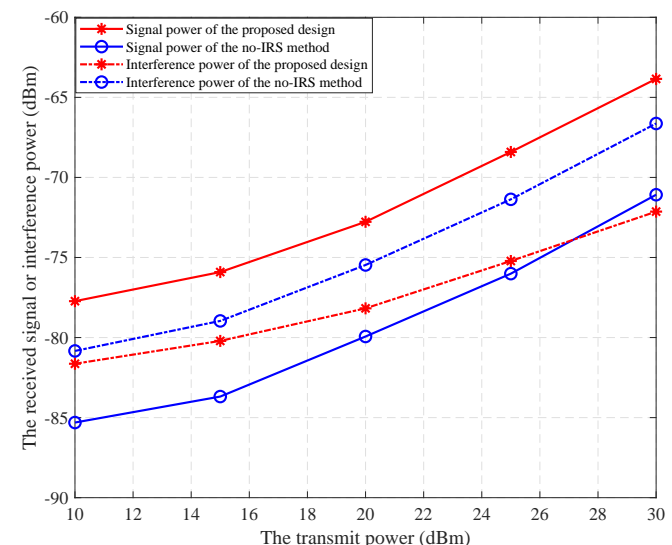

Fig. 9. The sum received signal or interference power at the Bobs versus the transmit power.

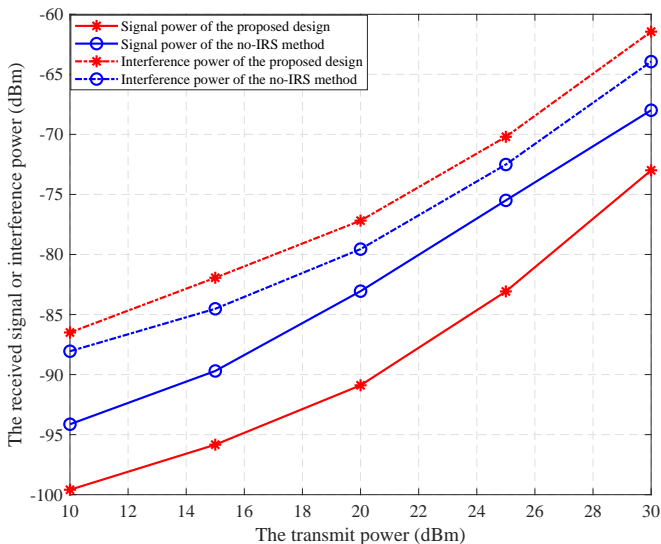

Fig. 10. The sum received signal or interference power at the Eves versus the transmit power.

sum received signal power and interference power over all Bob-Eve groups versus the transmit power budget $P_{T}$, in two different cases, e.g, with IRS and AN, with AN and without IRS. Firstly, from Fig. 9, we can see that with given transmit power, the signal power at the Bobs tends to increase in the IRS-aided case, while the interference power at the Bobs tends to decrease, when compared with the no-IRS-aided design. Then, from Fig. 10, we can see that with given transmit power, the signal power at the Eves tends to decrease, while the interference power at the Eves tends to increase, when compared with the no-IRS-aided design. Furthermore, from Fig. 9 and Fig. 10, we can see that the major effect of IRS is to enhance the signal power at the Bobs, while reduce the signal power at the Eves.

Nextly, we show the effect of the path loss exponent of the reflect link (i.e., the IRS-Bobs/Eves link) on the WSSR performance. Fig. 11 shows the WSSR versus the path loss exponent of the IRS-Bobs link. It can be seen from Fig. 11 that the WSSR decreases with the exponent, due to the fact that more severe fading leads to a weaker signal reflected from the IRS, thus reducing the benefits of the IRS. In addition, the WSSR versus the path loss exponent of the IRS-Eves link is shown in Fig. 12, where we can see that the WSSR increases with the exponent of the IRS-Eves link. This arises from the fact that weaker signal is reflected to Eves with the increase in the large-scale fading, thus degrading the reception at the Eves. These results reveal an insights that 


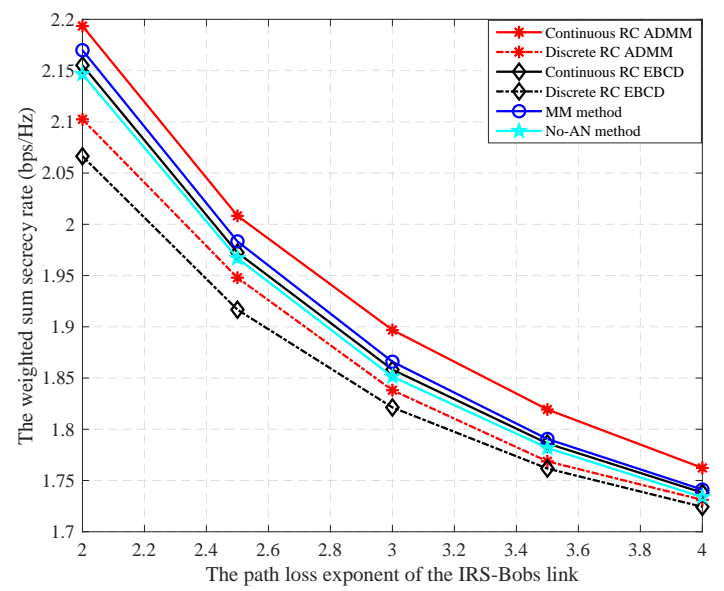

Fig. 11. The WSSR versus the path loss exponent of the IRS-

Bobs link.

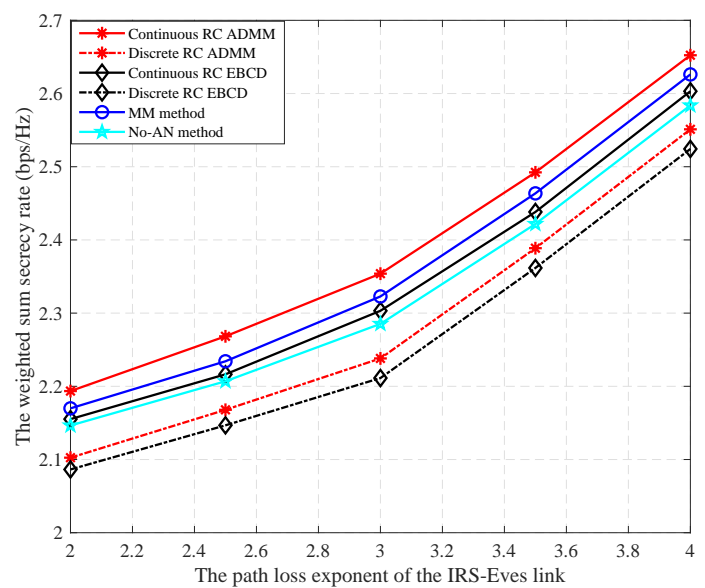

Fig. 12. The WSSR versus the path loss exponent of the IRSEves link.

the IRS should be properly deployed such that there are fewer blocking objects in the legitimate link or more blocking objects in the eavesdropping link to improve the secrecy performance.

To show the impact of the location of the IRS on the secrecy performance, Fig. 13 illustrates the obtained WSSR for these schemes versus different BS-IRS horizontal distances. It is observed that for all these IRS-aided methods, when IRS moves along the $x$-axis from the BS to the BobEve area (about $x_{\text {IRS }} \leq 50 \mathrm{~m}$ ), the WSSR first decreases than increases. When IRS moves away from the Bob-Eve area (about $x_{\text {IRS }}>50 \mathrm{~m}$ ), the WSSR decreases obviously due to the severe channel fading. In fact, for all IRS-aided schemes, the WSSR obtains its minimum value when the IRS is close to the middle between the BS and the center point of the Bob-Eve groups. Besides, when the IRS is neither close to the BS nor the Bobs, both the BS-IRS link and the IRS-Bobs links would experience significant attenuations that decrease the capability of the IRS in reflecting the incident signals to the Bobs. This result shows that it is beneficial for deploying the IRS close to BS or Bobs to enhance the security.

Lastly, to show the superiority of distributed IRS, we investigate a scenario where the BobEve groups are scattered in the corners of a larger square than that in Fig. 2. Specifically, BS is located at the center of the square with coordinate $(0 \mathrm{~m}, 0 \mathrm{~m})$, while the distance of each Bob to BS is set as $40 \mathrm{~m}$, respectively. In addition, the large IRS has 40 elements and each 


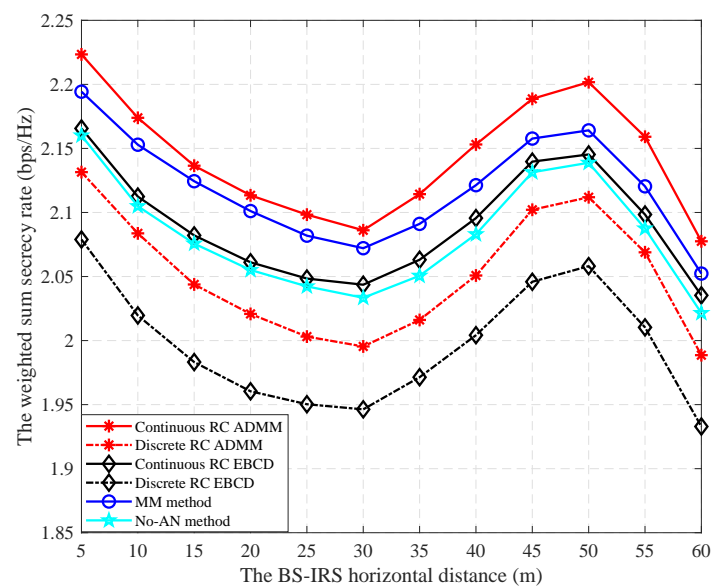

Fig. 13. The WSSR versus the BS-IRS horizontal distance.

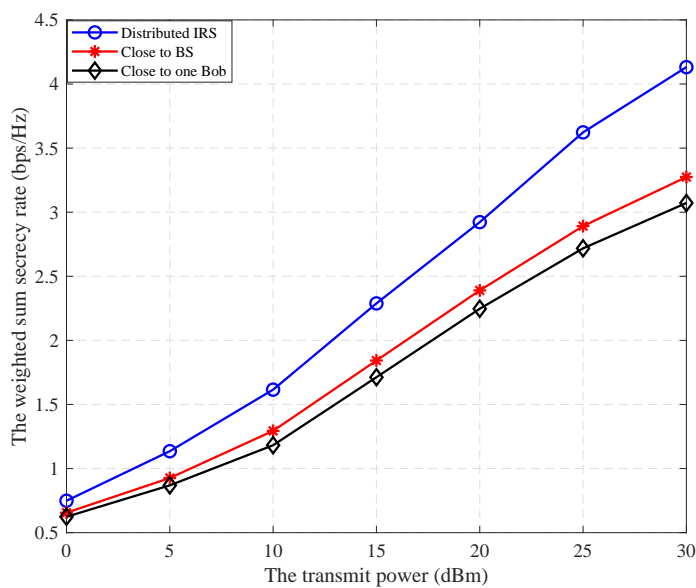

Fig. 14. The WSSR versus different IRS's deployments. distributed IRS has 10 elements. For the centralized IRS case, we consider two different IRS location designs, one is deployed at $(10 \mathrm{~m}, 10 \mathrm{~m})$, and the other is $(50 \mathrm{~m}, 50 \mathrm{~m})$, respectively, e.g., one is close to BS, the other one is close to one Bob. While for the distributed IRS case, similar to [32], we assume that the IRSs are scattered in the corners of a square with center at $\mathrm{BS}$, and the distance of each IRS to BS is set as $50 \mathrm{~m}$, thus, the distance between each IRS is more larger than the BS-IRS distance and the IRS-Bob distance. The three deployments are labelled as "Close to BS", "Close to one Bob", and "Distributed IRS", respectively, and the obtained WSSR is illustrated in Fig. 14. It is observed from the figure that, multiple smaller surfaces can improve the secrecy performance when each Bob-Eve group is relatively faraway from each other. This is mainly due to that when multiple IRSs are deployed in this network, the average link distance between each Bob and its nearest IRS is reduced. In addition, multiple IRSs create multiple independent propagation paths which introduces macro diversity, thus is beneficial to establish strong end-to-end links from BS to each Bob.

\section{Conclusion}

This paper investigated the use of IRS for MU-MISO secure transmission under the thread of multiple Eves. To evaluate the secrecy performance, we maximized the WSSR by jointly designing the secure BF, the AN jamming, and the RC of the IRS. Specifically, we developed 
an $\mathrm{AO}$ algorithm to handle the formulated non convex problem. The secure $\mathrm{BF}$ and the AN jamming matrix were optimally designed via the SCA method given the phase shift, which can be derived by utilizing an ADMM method or an EBCD method. Finally, simulation results were presented to demonstrate the benefits of the IRS to effectively improve the secrecy performance in comparison to other benchmark schemes.

\section{APPENDIX A}

\section{PROOF OF THEOREM 1.}

Firstly, during the update of $\left(\left\{\mathbf{w}_{k}\right\}_{k=1}^{K}, \mathbf{v}\right)$ at the $q+1$-th iteration, a upper bound $R_{s}^{u b 1}$ of the WSSR $R_{s}$ around given point $\left(\left\{\mathbf{w}_{k}^{q}\right\}_{k=1}^{K}, \mathbf{v}^{q}, \hat{\boldsymbol{\theta}}^{q}\right)$ is given by

$$
\begin{aligned}
& R_{s}^{u b 1}\left(\left\{\mathbf{w}_{k}\right\}_{k=1}^{K}, \mathbf{v}, \hat{\boldsymbol{\theta}}^{q}\right)= \\
& \sum_{k=1}^{K} \varphi_{k}\left\{\frac{2 \Re\left\{\mathbf{w}_{k}^{H} \tilde{\mathbf{H}}_{k}^{H} \hat{\boldsymbol{\theta}}^{q}\left(\hat{\boldsymbol{\theta}}^{q}\right)^{H} \tilde{\mathbf{H}}_{k} \mathbf{w}_{k}^{q}\right\}}{y_{k}^{q}}+\frac{2 \Re\left\{\left(\boldsymbol{\omega}_{k}^{q}\right)^{H} \boldsymbol{\Omega}_{k}^{H}\left(\mathbf{Q}_{k}^{q}\right)^{-1} \boldsymbol{\Omega}_{k} \boldsymbol{\omega}_{k}\right\}}{1-t_{k}^{q}}\right. \\
& \left.-\frac{\left(\boldsymbol{\omega}_{k}^{q}\right)^{H} \boldsymbol{\Omega}_{k}^{H}\left(\mathbf{Q}_{k}^{q}\right)^{-1} \mathbf{Q}_{k}\left(\mathbf{Q}_{k}^{q}\right)^{-1} \boldsymbol{\Omega}_{k} \boldsymbol{\omega}_{k}^{q}}{1-t_{k}^{q}}-\frac{\left|x_{k}^{q}\right|^{2}\left(y_{k}+\left|x_{k}\right|^{2}\right)}{y_{k}^{q}\left(y_{k}^{q}+\left|x_{k}^{q}\right|^{2}\right)}-\frac{z_{k}}{1+z_{k}^{q}}+\mathrm{C}\right\} .
\end{aligned}
$$

Similarly, in the update of $\hat{\boldsymbol{\theta}}$ at the $q+1$-th iteration, the following upper bound $R_{s}^{u b 2}$ of $R_{s}$ around given point $\left(\left\{\mathbf{w}_{k}^{q+1}\right\}_{k=1}^{K}, \mathbf{v}^{q+1}, \hat{\boldsymbol{\theta}}^{q}\right)$ can be obtained

$$
\begin{aligned}
& R_{s}^{u b 2}\left(\left\{\mathbf{w}_{k}^{q+1}\right\}_{k=1}^{K}, \mathbf{v}^{q+1}, \hat{\boldsymbol{\theta}}\right)= \\
& \sum_{k=1}^{K} \varphi_{k}\left\{\frac{2 \Re\left\{\hat{\boldsymbol{\theta}}^{H} \tilde{\mathbf{H}}_{k} \mathbf{w}_{k}^{q+1}\left(\mathbf{w}_{k}^{q+1}\right)^{H} \tilde{\mathbf{H}}_{k}^{H} \hat{\boldsymbol{\theta}}^{q}\right\}}{y_{k}^{q}}+\frac{2 \Re\left\{\hat{\boldsymbol{\theta}}^{H} \tilde{\mathbf{H}}_{e, k} \boldsymbol{\Psi}_{k}\left(\mathbf{P}_{k}^{q}\right)^{-1} \mathbf{\Psi}_{k}^{H} \tilde{\mathbf{H}}_{e, k}^{H} \hat{\boldsymbol{\theta}}^{q}\right\}}{1-u_{k}^{q}}\right. \\
& \left.-\frac{\left(\hat{\boldsymbol{\theta}}^{q}\right)^{H} \tilde{\mathbf{H}}_{e, k} \mathbf{\Psi}_{k}\left(\mathbf{P}_{k}^{q}\right)^{-1} \mathbf{P}_{k}\left(\mathbf{P}_{k}^{q}\right)^{-1} \mathbf{\Psi}_{k}^{H} \tilde{\mathbf{H}}_{e, k}^{H} \hat{\boldsymbol{\theta}}^{q}}{1-u_{k}^{q}}-\frac{\left|x_{k}^{q}\right|^{2}\left(y_{k}+\left|x_{k}\right|^{2}\right)}{y_{k}^{q}\left(y_{k}^{q}+\left|x_{k}^{q}\right|^{2}\right)}-\frac{z_{k}}{1+z_{k}^{q}}+\mathrm{C}\right\}
\end{aligned}
$$

With $R_{s}^{u b 1}\left(\left\{\mathbf{w}_{k}\right\}_{k=1}^{K}, \mathbf{v}, \hat{\boldsymbol{\theta}}^{q}\right)$ and $R_{s}^{u b 2}\left(\left\{\mathbf{w}_{k}^{q+1}\right\}_{k=1}^{K}, \mathbf{v}^{q+1}, \hat{\boldsymbol{\theta}}\right)$, we have

$$
\begin{aligned}
& R_{s}\left(\left\{\mathbf{w}_{k}^{q}\right\}_{k=1}^{K}, \mathbf{v}^{q}, \hat{\boldsymbol{\theta}}^{q}\right) \underset{(\mathrm{a})}{\leq} \max _{\left\{\mathbf{w}_{k}\right\}_{k=1}^{K}, \mathbf{v}} R_{s}^{u b 1}\left(\left\{\mathbf{w}_{k}\right\}_{k=1}^{K}, \mathbf{v}, \hat{\boldsymbol{\theta}}^{q}\right) \\
& \underset{(\mathrm{b})}{\leq} \max _{\hat{\boldsymbol{\theta}}} R_{s}^{u b 2}\left(\left\{\mathbf{w}_{k}^{q+1}\right\}_{k=1}^{K}, \mathbf{v}^{q+1}, \hat{\boldsymbol{\theta}}\right) \leq R_{s}\left(\left\{\mathbf{w}_{k}^{q+1}\right\}_{k=1}^{K}, \mathbf{v}^{q+1}, \hat{\boldsymbol{\theta}}^{q+1}\right),
\end{aligned}
$$


where (a) and (b) hold since $\left(\left\{\mathbf{w}_{k}^{q+1}\right\}_{k=1}^{K}, \mathbf{v}^{q+1}\right)$ and $\hat{\boldsymbol{\theta}}^{q+1}$ are the optimal solutions of the convex problems (14) and (21), respectively.

Furthermore, due to (5b) and (5c), we know that $\left(\left\{\mathbf{w}_{k}^{q}\right\}_{k=1}^{K}, \mathbf{v}^{q}\right)$ and $\hat{\boldsymbol{\theta}}^{q}$ are both bounded. According to the Cauchy's theorem [44], the sequence $\left(\left\{\mathbf{w}_{k}^{q}\right\}_{k=1}^{K}, \mathbf{v}^{q}, \hat{\boldsymbol{\theta}}^{q}\right)$ will converge to $\left(\left\{\mathbf{w}_{k}^{\star}\right\}_{k=1}^{K}, \mathbf{v}^{\star}, \hat{\boldsymbol{\theta}}^{\star}\right)$ as $q \rightarrow \infty$, i.e.,

$$
\begin{aligned}
& 0=\lim _{q \rightarrow \infty}\left\{R_{s}\left(\left\{\mathbf{w}_{k}^{q}\right\}_{k=1}^{K}, \mathbf{v}^{q}, \hat{\boldsymbol{\theta}}^{q}\right)-R_{s}\left(\left\{\mathbf{w}_{k}^{\star}\right\}_{k=1}^{K}, \mathbf{v}^{\star}, \hat{\boldsymbol{\theta}}^{\star}\right)\right\} \\
& \leq \lim _{q \rightarrow \infty}\left\{R_{s}\left(\left\{\mathbf{w}_{k}^{q+1}\right\}_{k=1}^{K}, \mathbf{v}^{q+1}, \hat{\boldsymbol{\theta}}^{q+1}\right)-R_{s}\left(\left\{\mathbf{w}_{k}^{\star}\right\}_{k=1}^{K}, \mathbf{v}^{\star}, \hat{\boldsymbol{\theta}}^{\star}\right)\right\} .
\end{aligned}
$$

Hence, we have proved that $R_{s}\left(\left\{\mathbf{w}_{k}^{q}\right\}_{k=1}^{K}, \mathbf{v}^{q}, \hat{\boldsymbol{\theta}}^{q}\right) \leq R_{s}\left(\left\{\mathbf{w}_{k}^{q+1}\right\}_{k=1}^{K}, \mathbf{v}^{q+1}, \hat{\boldsymbol{\theta}}^{q+1}\right)$, which can guarantee to converge to a locally optimal point.

\section{APPENDIX B}

\section{Proof of TheOREM 2.}

As mentioned, the sequence $\left(\left\{\mathbf{w}_{k}^{q}\right\}_{k=1}^{K}, \mathbf{v}^{q}, \hat{\boldsymbol{\theta}}^{q}\right)$ will converges to $\left(\left\{\mathbf{w}_{k}^{\star}\right\}_{k=1}^{K}, \mathbf{v}^{\star}, \hat{\boldsymbol{\theta}}^{\star}\right)$ as $q \rightarrow$ $\infty$. Next, we write the Lagrangian function of (14) as

$$
\mathcal{L}_{1}\left(\left\{\mathbf{w}_{k}\right\}_{k=1}^{K}, \mathbf{v}, \hat{\boldsymbol{\theta}}^{q}, \lambda\right)=R_{s}^{u b 1}\left(\left\{\mathbf{w}_{k}\right\}_{k=1}^{K}, \mathbf{v}, \hat{\boldsymbol{\theta}}^{q}\right)+\lambda\left(\sum_{k=1}^{K}\left\|\mathbf{w}_{k}\right\|^{2}+\|\mathbf{v}\|^{2}-P_{s}\right),
$$

where $\lambda \geq 0$ is the dual variables for $(5 b)$.

Then, when $q \rightarrow \infty$, the related KKT conditions are given as follows: $\nabla_{\mathbf{w}_{k}} R_{s}^{u b 1}\left(\left\{\mathbf{w}_{k}^{\star}\right\}_{k=1}^{K}, \mathbf{v}^{\star}, \hat{\boldsymbol{\theta}}^{q}\right)+$ $2 \lambda^{\star} \mathbf{w}_{k}^{\star}=\mathbf{0}, \forall k, \nabla_{\mathbf{v}} R_{s}^{u b 1}\left(\left\{\mathbf{w}_{k}^{\star}\right\}_{k=1}^{K}, \mathbf{v}^{\star}, \hat{\boldsymbol{\theta}}^{q}\right)+2 \lambda^{\star} \mathbf{v}^{\star}=\mathbf{0}$, and $\lambda^{\star}\left(\sum_{k=1}^{K}\left\|\mathbf{w}_{k}^{\star}\right\|^{2}+\left\|\mathbf{v}^{\star}\right\|^{2}-P_{s}\right)=$ 0.

Similarly, the Lagrangian function of (19) is

$$
\mathcal{L}_{2}\left(\left\{\mathbf{w}_{k}^{q}\right\}_{k=1}^{K}, \mathbf{v}^{q}, \hat{\boldsymbol{\theta}}, \boldsymbol{\eta}\right)=R_{s}^{u b 2}\left(\left\{\mathbf{w}_{k}^{q}\right\}_{k=1}^{K}, \mathbf{v}^{q}, \hat{\boldsymbol{\theta}}\right)+\sum_{m=1}^{M} \eta_{m}\left(\hat{\theta}_{m}^{\dagger} \hat{\theta}_{m}-1\right),
$$

where $\boldsymbol{\eta}=\left[\eta_{1} \geq 0, \ldots, \eta_{M} \geq 0\right] \in \mathbb{R}^{1 \times M}$ is the dual variable for the unit modulus constraint. Then, the KKT condition for $\hat{\boldsymbol{\theta}}$ is given by $\nabla_{\hat{\theta}_{m}} R_{s}^{u b 2}\left(\left\{\mathbf{w}_{k}^{q}\right\}_{k=1}^{K}, \mathbf{v}^{q}, \hat{\boldsymbol{\theta}}^{\star}\right)+\eta_{m}^{\star} \hat{\theta}_{m}^{\star}=0, \forall m$, and $\eta_{m}^{\star}\left(\left(\hat{\theta}_{m}^{\star}\right)^{\dagger} \hat{\theta}_{m}^{\star}-1\right)=0, \forall m$. Since $\left(\left\{\mathbf{w}_{k}^{\star}\right\}_{k=1}^{K}, \mathbf{v}^{\star}\right)$ and $\hat{\boldsymbol{\theta}}^{\star}$ are the optimal solutions of (14) and (21), respectively, thus the above KKT conditions are satisfied. Hence, the converged solution $\left(\left\{\mathbf{w}_{k}^{\star}\right\}_{k=1}^{K}, \mathbf{v}^{\star}, \hat{\boldsymbol{\theta}}^{\star}\right)$ is a KKT point. 


\section{REFERENCES}

[1] Q. Wu, S. Zhang, B. Zheng, C. You, and R. Zhang, "Intelligent reflecting surface aided wireless communications: A tutorial," IEEE Trans. Commun., doi: 10.1109/TCOMM.2021.3051897.

[2] J. Lyu, and R. Zhang, "Spatial throughput characterization for intelligent reflecting surface aided multiuser system," IEEE Wireless Commun. Lett., vol. 9, no. 6, pp. 834-838, Jun. 2020.

[3] W. Yan, X. Yuan, Z.-Q. He, and X. Kuai, "Passive beamforming and information transfer design for reconfigurable intelligent surfaces aided multiuser MIMO systems," IEEE J. Sel. Areas Commun., vol. 38, no. 8, pp. 1793-1808, Aug. 2020.

[4] S. Hu, F. Rusek, and O. Edfors, "Beyond massive MIMO: The potential of data transmission with large intelligent surfaces," IEEE Trans. Signal Process., vol. 66, no. 10, pp. 2746-2758, May 2018.

[5] W. Tang, M. Chen, J. Dai, Y. Zeng, X. Zhao, S. Jin, Q. Cheng, and T. Cui, "Wireless communications with programmable metasurface new paradigms, opportunities, and challenges on transceiver design," IEEE Wireless Commun., vol. 27, no. 2, pp. 180-187, Apr. 2020.

[6] Q. Wu and R. Zhang, “Towards smart and reconfigurable environment: Intelligent reflecting surface aided wireless networks,” IEEE Commun. Mag., vol. 58, no. 1, pp. 106-112, Jan. 2020.

[7] S. Gong, X. Lu, D. Hoang, D. Niyato, L. Shu, D. Kim, and Y. C. Liang, "Toward smart wireless communications via intelligent reflecting surfaces: A contemporary survey," IEEE Commun. Surveys Tut., vol. 22, no. 4, pp. 2283-2314, 4th Quart. 2020.

[8] Q. Wu and R. Zhang, "Intelligent reflecting surface enhanced wireless network via joint active and passive beamforming," IEEE Trans. Wireless Commun., vol. 18, no. 11, pp. 5394-5409, Nov. 2019.

[9] Q. Wu, and R. Zhang, "Joint active and passive beamforming optimization for intelligent reflecting surface assisted SWIPT under QoS constraints," IEEE J. Sel. Areas Commun., vol. 38, no. 8, pp. 1735-1748, Aug. 2020.

[10] H. Guo, Y. Liang, J. Chen, and E. G. Larsson, "Weighted sum-rate maximization for reconfigurable intelligent surface aided wireless networks," IEEE Trans. Wireless Commun., vol. 19, no. 5, pp. 3064-3076, May. 2020.

[11] G. Zhou, C. Pan, H. Ren, K. Wang, and A. Nallanathan, "A framework of robust transmission design for IRS-aided MISO communications with imperfect cascaded channels," IEEE Trans. Signal Process., vol. 68, pp. 5092-5106, Aug. 2020.

[12] Q. Wu and R. Zhang, "Beamforming optimization for wireless network aided by intelligent reflecting surface with discrete phase shifts," IEEE Trans. Commun., vol. 68, no. 3, pp. 1838-1851, Mar. 2020.

[13] H. Shen, W. Xu, S. Gong, C. Zhao, and D. W. K. Ng, "Beamforming optimization for IRS-aided communications with transceiver hardware impairments," IEEE Trans. Commun., vol. 69, no. 2, pp. 1214-1227, Feb. 2021.

[14] C. Pan, H. Ren, K. Wang, M. Elkashlan, A. Nallanathan, J. Wang, and L. Hanzo, "Intelligent reflecting surface aided MIMO broadcasting for simultaneous wireless information and power transfer," IEEE J. Sel. Areas Commun., vol. 38, no. 8, pp. 1719-1734, Aug. 2020. 
[15] G. Zhou, C. Pan, H. Ren, K. Wang, and A. Nallanathan, "Intelligent reflecting surface aided multigroup multicast MISO communication systems," IEEE Trans. Signal Process., vol. 68, pp. 3236-3251, Apr. 2020.

[16] C. Pan, H. Ren, K. Wang, W. Xu, M. Elkashlan, A. Nallanathan, and L. Hanzo, "Multicell MIMO communications relying on intelligent reflecting surface," IEEE Trans. Wireless Commun., vol. 19, no. 8, pp. 5218-5233, Aug. 2020.

[17] C. Huang, R. Mo and C. Yuen, "Reconfigurable intelligent surface assisted multiuser MISO systems exploiting deep reinforcement learning," IEEE J. Sel. Areas Commun., vol. 38, no. 8, pp. 1839-1850, Aug. 2020.

[18] J. Yuan, Y. C. Liang, J. Joung, G. Feng, and E. G. Larsson, "Intelligent reflecting surface-assisted cognitive radio system," IEEE Trans. Commun., vol. 69, no. 1, pp. 675-687, Jan. 2021.

[19] Y. Zou, S. Gong, J. Xu, W. Cheng, D. T. Hoang and D. Niyato, "Wireless powered intelligent reflecting surfaces for enhancing wireless communications,” IEEE Trans. Vec. Tech., vol. 69, no. 10, pp. 12369-12373, Oct. 2020.

[20] H. Shen, T. Ding, W. Xu, and C. Zhao, "Beamforming design with fast convergence for IRS-aided full-duplex communication," IEEE Commun. Lett., vol. 24, no. 12, pp. 2849-2853, Dec. 2020.

[21] J. Zuo, Y. Liu, Z. Qin, and N. A.-Dhahir, "Resource allocation in intelligent reflecting surface assisted NOMA systems," IEEE Trans. Commun., vol. 68, no. 11, pp. 7170-7183, Nov. 2020.

[22] F. Shu, J. Li, M. Huang, W. Shi, Y. Teng, J. Li, Y. Wu, and J. Wang, "Enhanced secrecy rate maximization for directional modulation networks via IRS,” 2020, arXiv: 2008.05067v1, [Online]. Available: https://arxiv.org/abs/2008.05067.

[23] Y. Han, S. Zhang, L. Duan, and R. Zhang, "Cooperative double-IRS aided communication beamforming design and power scaling," IEEE Wireless Commun. Lett., vol. 9, no. 8, pp. 1206-1210, Aug. 2020.

[24] C. You, B. Zheng, and R. Zhang, "Wireless communication via double IRS: Channel estimation and passive beamforming designs," IEEE Wireless Commun. Lett., vol. 10, no. 2, pp. 431-435, Feb. 2021.

[25] W. Mei and R. Zhang, "Cooperative beam routing for multi-IRS aided communication," IEEE Wireless Commun. Lett., vol. 10, no. 2, pp. 426-430, Feb. 2021.

[26] B. Zheng, C. You, and R. Zhang, "Double-IRS assisted multi-user MIMO: Cooperative passive beamforming design," IEEE Trans. Wireless. Commun., doi: 10.1109/TWC.2021.3059945.

[27] Y. Wu, A. Khisti, C. Xiao, G. Caire, K. K. Wong, and X. Gao, "A survey of physical layer security techniques for 5G wireless networks and challenges ahead," IEEE J. Sel. Areas Commun., vol. 36, no. 4, pp. 679-695, Apr. 2018.

[28] J. Chen, Y. Liang, Y. Pei, and H. Guo, "Intelligent reflecting surface: A programmable wireless environment for physical layer security," IEEE Access, vol. 7, pp. 82599-82612, Jun. 2019.

[29] H. Shen, W. Xu, S. Gong, Z. He, and C. Zhao, "Secrecy rate maximization for intelligent reflecting surface assisted multi-antenna communications," IEEE Commun. Lett., vol. 23, no. 9, pp. 1488-1492, Sep. 2019.

[30] Z. Chu, W. Hao, P. Xiao, and J. Shi, "Intelligent reflecting surface aided multi-antenna secure transmission," IEEE Wireless Commun. Lett., vol. 9, no. 1, pp. 108-112, Jan. 2020.

[31] L. Dong and H.-M. Wang, "Secure MIMO transmission via intelligent reflecting surface," IEEE Wireless Commun. Lett., vol. 9, no. 6, pp. 787-790, Jan. 2020. 
[32] X. Yu, D. Xu, Y. Sun, D. W. K. Ng, and R. Schober, "Robust and secure wireless communications via intelligent reflecting surfaces," IEEE J. Sel. Areas Commun., vol. 38, no. 11, pp. 2637-2652, Nov. 2020.

[33] S. Hong, C. Pan, H. Ren, K. Wang, and A. Nallanathan, "Artificial-noise-aided secure MIMO wireless communications via intelligent reflecting surface," IEEE Trans. Commun., vol. 68, no. 12, pp. 7851-7866, Dec. 2020.

[34] Z. Chu, W. Hao, P. Xiao, D. Mi, Z. Liu, M. Khalily, J. R. Kelly, and A. P. Feresidis, "Secrecy rate optimization for intelligent reflecting surface assisted MIMO system,” IEEE Trans. Inf. Forensics Security, vol. 16, pp. 1655-1669, Nov. 2020

[35] B. Feng, Y. Wu, M. Zheng, X. Xia, Y. Wang, and C. Xiao, "Large intelligent surface aided physical layer security transmission,” IEEE Trans. Signal Process., vol. 68, pp. 5276-5291, Sep. 2020.

[36] H. Wang, J. Bai, and L. Dong, "Intelligent reflecting surfaces assisted secure transmission without eavesdropper's CSI," IEEE Signal Process. Lett., vol. 27, pp. 1300-1304, Jul. 2020.

[37] W. Shi, J. Li, G. Xia, Y. Wang, X. Zhou, Y. Zhang, F. Shu, "Secure multigroup multicast communication systems via intelligent reflecting surface," China Commun., vol. 18, no. 3, pp. 39-51, Mar. 2021.

[38] L. Dong, H. Wang, J. Bai, and H. Xiao, "Double intelligent reflecting surface for secure transmission with inter-surface signal reflection,” IEEE Trans. Veh. Tech., vol. 70, no. 3, pp. 2912-2916, Mar. 2021.

[39] S. Abeywickrama, R. Zhang, Q. Wu, and C. Yuen, "Intelligent reflecting surface: Practical phase shift model and beamforming optimization," IEEE Trans. Commun., vol. 68, no. 9, pp. 5849-5863, Sep. 2020.

[40] Q. Li, C. Li, and J. Lin, "Constant modulus secure beamforming for multicast massive MIMO wiretap channels," IEEE Trans. Inf. Forensics Security, vol. 15, pp. 264-275, Jul. 2020.

[41] H. Guo, Y. Liang, J. Chen, and E. G. Larsson, "Weighted sum-rate optimization for intelligent reflecting surface enhanced wireless networks," 2019, arXiv: 1905.07920v2, [Online]. Available: https://arxiv.org/abs/1905.07920.

[42] Z. Wang, L. Liu, and S. Cui, "Channel estimation for intelligent reflecting surface assisted multiuser communications: Framework, algorithms, and analysis," IEEE Trans. Wireless Commun., vol. 19, no. 10, pp. 6607-6620, Oct. 2020.

[43] P. Wang, J. Fang, H. Duan, and H. Li, “Compressed channel estimation for intelligent reflecting surface assisted millimeter wave systems," IEEE Signal Process. Lett., vol. 27, pp. 905-909, May 2020.

[44] A. A. Nasir, H. D. Tuan, T. Q. Duong, and H. V. Poor, "Secrecy rate beamforming for multicell networks with information and energy harvesting," IEEE Trans. Signal Process., vol. 65, no. 3, pp. 677-689, Feb. 2017.

[45] M. Grant and S. Boyd, CVX: Matlab software for disciplined convex programming, version 2.0 beta, Sep. 2012. Available: http://cvxr.com/cvx. 